famer bis Shale m

James H. Thrall, M.D., received his undergraduate and medical degrees at the University of Michigan. He then completed a rotating internship and residency training in Radiology at Walter Reed Army Medical Center in Washington, D.C., followed by a fellowship in nuclear medicine and two years as Assistant Chief of Nuclear Medicine, also at Walter Reed. Dr. Thrall joined the faculty of the University of Michigan Medical School in 1975 and served for 8 years until being appointed Chairman of the Department of Diagnostic Radiology at Henry Ford Hospital in Detroit in 1983. Dr. Thrall is currently Radiologist-in-Chief at the Massachusetts General Hospital and Professor of Radiology at the Harvard Medical School.

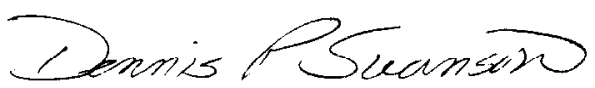

Dennis P. Swanson, R.Ph., M.S., is a board certified (A.Ph.A./B.P.S.) nuclear pharmacist in charge of the Division of Radiology Pharmacy Services, Department of Diagnostic Radiology, Henry Ford Hospital, Detroit, Michigan. Mr. Swanson also holds the academic appointments of Clinical Associate Professor of Pharmacy, College of Pharmacy, University of Michigan, and Adjunct Associate Professor of Pharmacy Practice, College of Pharmacy and Allied Health Professions, Wayne State University. He received his B.S. degree in pharmacy from the University of Iowa in 1971 and M.S. degree in nuclear pharmacy from the University of Southern California in 1976. Mr. Swanson has authored/coauthored numerous articles, book chapters, and abstracts related to the clinical use of radiopharmaceuticals and radiopaque contrast media. He has also served as a member of several committees within the Society of Nuclear Medicine, the American Society of Hospital Pharmacy, and the American Pharmaceutical Association. 


\title{
DIAGNOSTIC INTERVENTIONS IN NUCLEAR MEDICINE
}

\begin{abstract}
Diagnostic interventions in nuclear medicine may be defined as the coadministration of a nonradioactive drug or application of a physical stimulus or physiologic maneuver to enhance the diagnostic utility of a nuclear medicine test. The rationale for each interventional maneuver follows from the physiology or metabolism of the particular organ or organ system under evaluation. Diagnostic inference is drawn from the pattern of change in the biodistribution of the tracer in response to the intervention-induced change in metabolism or function.
\end{abstract}

In current practice, the most commonly performed interventional maneuvers are aimed at studies of the heart, genitourinary system, hepatobiliary system, and gastrointestinal tract. The single most commonly performed interventional study in the United States is the stress Thallium-201 myocardial perfusion scan aimed at the diagnosis of coronary artery disease. The stress portion of the study is accomplished with dynamic leg exercise on a treadmill and is aimed at increasing myocardial oxygen demands. Areas of myocardium distal to hemodynamically significant lesions in the coronary arteries become ischemic at peak stress due to the inability of the stenotic vessel to respond to the oxygen demand/blood flow needs of the myocardium. Ischemic areas are readily recognized as photopenic defects on scans obtained immediately after exercise, with "normalization" upon delayed imaging.

Diuresis renography is aimed at the differential diagnosis of hydroureteronephrosis. By challenging the urinary tract collecting structures with an augmented urine flow, dilated, unobstructed systems can be differentiated from systems with significant mechanical obstruction. Obstructed systems have a low ability to respond even after effective diuresis, resulting in a characteristic prolonged retention of the radiotracer.

Hepatobiliary interventions are most commonly employed in the clinical setting of suspected acute cholecystitis. Administering a cholecystogogue before a hepatobiliary tracer promotes visualization of the gallbladder by causing it to go through a contraction/filling cycle in which the filling phase occurs during maximum exposure to the radionuclide. This maneuver can convert a false positive study that suggests 
the presence of acute cholecystitis to a true negative study. Other gastrointestinal interventions are aimed at enhancing the detection of gastroesophageal reflux and gastrointestinal bleeding.

Many new interventions have been developed that are currently aimed at research problems rather than clinical problems. Elegant studies eliciting cortical activation in response to visual, auditory, and cognitive stimulae have been described for the brain and show clinical promise for the future. New interventions are also under investigation for the heart and kidney. The development of new tracers and instrumentation will continue to be paralleled by the development of new interventional maneuvers. 


\section{DIAGNOSTIC INTERVENTIONS IN NUCLEAR MEDICINE}

Over the past decade, interventional maneuvers have become an integral part of diagnostic nuclear medicine.$^{1-3}$ Within this context, interventions may be defined as the coadministration of a drug or the application of a physiological or physical stimulus to enhance or extend the diagnostic capability of a nuclear medicine examination. Although diagnostic interventions have only been used extensively in the last several years, they were applied early in the history of this specialty. Soon after the availability of ${ }^{131} \mathrm{I}$ for medical use, it was recognized that the percent localization of the tracer in the thyroid was a useful marker of specific organ function. The thyroid glands of hyperthyroid subjects demonstrated greater than normal accumulation; conversely, hypothyroid subjects typically had decreased percent uptakes. By performing the radioiodine percent uptake measurement in conjunction with interventional stimulation or suppression of the thyroid gland, additional useful information on thyroid autonomy and functional reserve, respectively, could be obtained. Such interventional maneuvers with ${ }^{131}$ I were described and well established during the late 1940s and early 1950s.

The early thyroid interventional studies have remained useful but have never represented a large portion of nuclear medicine practice. Although waterloading and mannitol infusion enjoyed brief vogues in renal studies aimed at detecting renal vascular hypertension, little was added to the interventional armamentarium during the 1950s and 1960s.

In the 1970s, a number of developments encouraged the growth and diversification of interventional techniques. Nuclear medicine computer systems became widely available and permitted the quantitative analysis of radiotracer distribution and, of equal importance, dynamic function studies of the time-activity distribution of radiotracers. This new analytic capability was coupled with the development of new radiopharmaceuticals and new applications of existing radiopharmaceuticals in virtually every organ system of the body. As a result, it is not uncommon to find that $25 \%$ to $50 \%$ of all studies in a full-service nuclear medicine department involve an interventional maneuver.

The wide variety of interventional nuclear medicine procedures makes it difficult to provide a simple classification. The essential purpose of an intervention is to alter organ function and gain diagnostic information, either by noting a change in the biodistribution of the radiopharmaceutical or by directly measuring a change in organ function with a radiotracer technique. ${ }^{1}$ For example, the radioiodine percent uptake test is essentially a measurement of the relative biodistribution of radioiodine at a fixed time within the thyroid gland. Diagnostic inference is achieved by obtaining a baseline measurement and noting whether a change occurs following the intervention, i.e., whether there is a change in the biodistribution. On the other hand, in a stress radionuclide ventriculogram, the biodistribution of the radiopharmaceutical does not change. The radiolabeled red blood cells remain within the vascular space, but changes in ventricular ejection fraction induced by the intervention are measured by using the radiotracer.

In addition to the use of interventions for the cnhancement of nuclear diagnoses, an important correlate has also evolved: the assessment of drug 
efficacy (or toxicity) by using radionuclide techniques. For example, in a patient with an established diagnosis of impaired left ventricular function due to coronary artery disease, the benefits of cardiac drugs are readily determined by nuclear medicine assessments of ventricular function performed before and after drug administration. Another example involves the administration of metoclopramide to promote gastric emptying in diabetics with gastric paresis. Response to the drug is variable and requires some residual innervation of the stomach. The use of radionuclide techniques to directly measure the gastric emptying response after metoclopramide administration provides an objective rationale for administering or not administering the drug, an important consideration in view of its significant potential side effects.

From the preceding discussion, it becomes apparent that each interventional maneuver has a specific rationale that must be met if the intervention is to be meaningful. For pharmacological interventions, a dose of the drug must be determined that will reliably achieve the desired or critical change in organ function. For example, if too little thyroid hormone is administered in determining thyroid autonomy, the test results will obviously be invalid. Dosage regimens have been established in the development of each pharmacological intervention to reliably achieve the desired objective. Physiological and physical interventions are more challenging in this respect, but in each case, criteria have been developed to permit assessment of the adequacy of the intervention. In cardiac stress testing, the heart rate and blood pressure response to exercise are measured as indicators of the work being performed by the heart and, therefore, the adequacy of the intervention. The development of better methods to independently determine whether the rationale for a specific interventional maneuver has been met continues to be a challenge. Likewise, to compare results between patients, protocols must be reproducible. For pharmacological interventions, this process is reasonably straightforward, but in interventions requiring patient involvement and compliance, reproducibility is more problematic.

Literally dozens of interventional techniques have been described in the literature. This article will not attempt to summarize all of them but will emphasize primarily those procedures and techniques that have achieved widespread clinical acceptance and are in routine daily use (Table 1 ). Some additional interventions will be described to illustrate developmental trends and the wealth of opportunity for future clinical studies.
TABLE 1.

Clinically Important Diagnostic Interventions

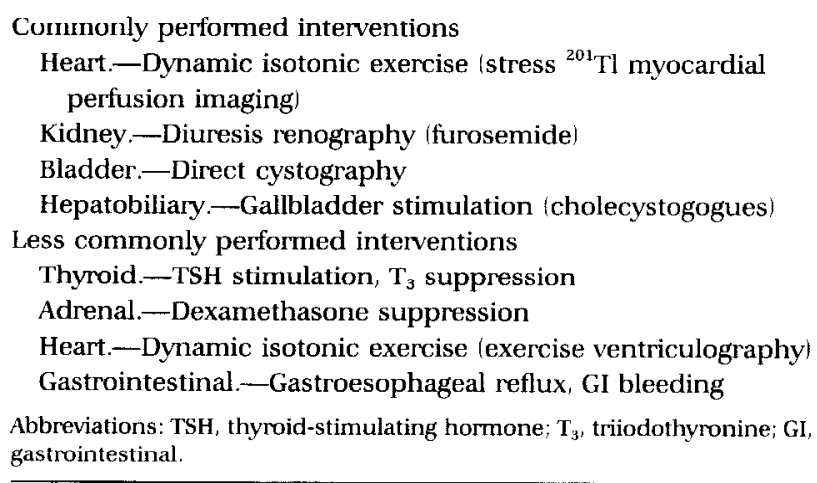

\section{THYROID INTERVENTIONS}

As noted in the introduction, interventional studies of the thyroid gland were the first to be established in nuclear medicine practice. Werner and Spooner demonstrated that thyroidal uptake of iodine would suppress to less than $50 \%$ of baseline values after administration of thyroid hormone. ${ }^{4}$ Thyroid suppression testing became valuable for distinguishing hyperthyroid from euthyroid patients in selected cases and for detecting patients with euthyroid Graves' disease. The primary disadvantage of the suppression test is that it requires two radioiodine uptake measurements 1 week apart and the administration of exogenous thyroid hormone to patients frequently already thyrotoxic. ${ }^{5}$ In current practice, the suppression test has given way to the thyrotropinreleasing hormone infusion test, which measures the thyroid-stimulating hormone response to thyrotropin-releasing hormone administration. This test can be performed in an hour and requires no exposure of the patient to ionizing radiation or thyroid hormone. ${ }^{6,7}$

The protocol most frequently used for the thyroid suppression test includes a baseline 24-hour radioiodine uptake measurement followed by the administration of triiodothyronine, $25 \mu \mathrm{g}$ four times a day for 8 days, followed by a second 24hour radioiodine uptake test on the last day of the suppression regimen. Failure of the uptake to decrease by more than $\mathbf{5 0} \%$ from baseline values is considered abnormal.

The suppression test has also been adapted to the evaluation of functioning thyroid nodules to determine whether they are autonomous. ${ }^{8,9}$ After a baseline scan, thyroid function is suppressed by using the triiodothyronine regimen outlined earlier with a follow-up scan obtained on the last day of the test. If the functioning nodule is the only portion of the thyroid visualized, autonomy or the 
lack thereof can be established by the quantitative criteria cited for the suppression test. If both nodular and non-nodular activity are visualized, an assessment must be made of the relative suppressibility of the nodular activity in relation to the rest of the gland. If the extranodular tissue activity is clearly suppressed without change in uptake in the nodule, autonomous function is inferred. If nodular activity is suppressed to an equal degree and the overall uptake declines by more than $50 \%$, the inference is that the nodule is not autonomous. If multiple functioning nodules are present or the response is not clearly defined, it may be difficult to prove or disprove the autonomy of a given nodule.

Clinically, thyroid hormone therapy is often used to reduce the size of benign goiters and thyroid nodules. If suppression testing reveals nonsuppressibility, the nodule will probably not respond to thyroid hormone therapy and must be dealt with in some other manner. Although the triiodothyronine suppression test is usually well tolerated, it is contraindicated in patients with heart disease. If their thyroid function is not suppressible, the added exogenous hormone may result in cardiac toxicity, including tachyarrhythmias or angina.

The thyroid-stimulating hormone stimulation test was originally used in conjunction with the radioiodine percent uptake test to measure thyroid reserve. It has also been used in conjunction with imaging to diagnose congenital thyroid hemiagenesis, to determine the extent of extranodular thyroid tissue in patients with autonomous functioning nodules, and to enhance tracer localization in follow-up scanning for metastatic disease after thyroid surgery for cancer. ${ }^{10}$

In normal subjects and patients with hypopituitarism, the percent uptake ordinarily doubles after 5 to 10 units of thyroid stimulating hormone are administered intramuscularly for 3 days. ${ }^{5}$ Patients with primary hypothyroidism demonstrate diminished functional reserve. Diminished reserve is also seen in some patients with nontoxic multinodular goiters and Hashimoto's thyroiditis.

The thyroid-stimulating hormone stimulation test is only used occasionally in current practice. The differentiation of primary and secondary hypothyroidism is now accomplished by the thyroidreleasing hormone-thyroid-stimulating hormone test. Moreover, there is some risk in administering thyroid-stimulating hormone since the clinically available agent is a bovine protein ${ }^{11,12}$ Antigen-antibody reactions can occur, especially with repeated studies. ${ }^{12,13}$ In one series, allergic reactions of varying severity were observed in $43 \%$ of pa- tients receiving repeat doses of thyroid-stimulating hormone. ${ }^{13}$

A third interventional technique involving the radioiodine percent uptake procedure is the perchlorate discharge test. ${ }^{14}$ This test measures the dissociation between trapping and organification of iodine in the thyroid gland. Radioiodine is administered to the patient, after which a baseline percent uptake measurement is taken at 1 to 2 hours. One gram of potassium perchlorate is administered orally, and follow-up measurements are made hourly. In normal subjects and in hyperthyroid patients receiving inadequate antithyroid drug therapy, there is less than a $\mathbf{1 0 \%}$ decline in percent uptake from the baseline value. Dissociation of trapping and organification is indicated by a decrease greater than $10 \%$. Patients with Hashimoto's thyroiditis or peroxidase erizyme deficiency and patients receiving inadequate antithyroid drug therapy for hyperthyroidism demonstrate this dissociation. ${ }^{15,16}$

\section{ADRENAI, INTERVENTIONS}

The advent of computed tomography (CT) shortly after the clinical introduction of radiolabeled cholesterol derivatives for adrenal cortical scintigraphy eroded much of the interest in the nuclear medicine technique. Clinically, it is important to determine whether the cause of excess adrenal hormone production is due to an adrenal adenoma or hyperplasia. If adenoma is present, the therapy is often unilateral adrenalectomy. If hyperplasia occurs, the therapy may be directed either at the pituitary, a paraendocrine source of stimulating hormone, or in cases of primary hyperplasia at the adrenals themselves. If an adenoma is detected by CT scanning, the workup may be complete; if not, adrenal scintigraphy may still play a role.

For cortisol-producing lesions, the incorporation of dexamethasone suppression into the adrenal cortical scintigraphy procedure is not necessary because of the adrenal-pituitary feedback mechanisms. In cortisol-producing adenomas, the excess hormone suppresses pituitary adrenocorticotropic hormone (ACTH) release, and as a result, the contralateral normal adrenal does not accumulate the radiotracer. ${ }^{17,18}$ Scintigraphy reveals intense unilateral activity corresponding to the adenoma. In cases of bilateral hyperplasia, both adrenals are visualized.

In patients with hyperaldosteronism or hyperandrogenism, the excess hormone does not cause feedback suppression of pituitary ACTH release. It is therefore often difficult to distinguish adenoma 
from hyperplasia without suppression testing (Fig 1). ${ }^{19-24}$ The suppression test involves the oral administration of dexamethasone, $4 \mathrm{mg} / \mathrm{day}$, in divided doses for 7 days prior to the administration of radiocholesterol. Dexamethasone administration is continued throughout the imaging interval. $^{20,22}$ With this interventional regimen, the normal adrenal cortices should not be visualized until at least 5 days after radiotracer injection. The presence of an aldosteronoma or androgenoma is indicated by unilateral or asymmetrical uptake on images obtained 1 or 2 days after tracer administration. In patients with bilateral hyperplasia, early (i.e., within 5 days after radiocholesterol administration) bilateral visualization is typically demonstrated. $^{22-24}$ The technique is reasonably accurate. Freitas et al. reported a series of 20 patients with hyperaldosteronism in whom the scintigraphic technique correctly located the site of nine of ten adenomas and distinguished adenoma from hyperplasia in $90 \%$ of the cases. ${ }^{23}$

\section{RENAL INTERVENTIONS}

Two quite different renal interventions have become clinically important. Diuresis renography is aimed at the differential diagnosis of causes of pyelocaliceal and ureteral dilation, and radionuclide cystography is aimed at detecting vesicoureteral reflux.

\section{DIURESIS RENOGRAPHY}

The management of mechanical obstruction of the ureteropelvic junction or ureter is usually surgical, while medical management is often appropriate for a dilated, but nonobstructed collecting system. Conventional urography with intravenous radiographic contrast media and conventional radionuclide renography do not reliably differentiate cases of obstructive vs. nonobstructive hydroureteronephrosis because the findings are not specific. The dilation, delayed opacification, and delayed washout typical of the obstructive conditions may also be seen in many other conditions that cause collecting system dilation but are not necessarily associated with mechanical obstruction (Fig 2).

In attempting to determine the significance of collecting system dilation two different approaches have been developed to assess urodynamics. In 1973, Whittaker described a percutaneous ureteral perfusion technique to measure pressure-flow relationships in the renal pelvis. ${ }^{25} \mathrm{~A}$ catheter or trocar is placed in the renal pelvis, and the bladder is catheterized. A baseline measurement of the pressures in the renal pelvis and bladder is obtained, and pressures are continuously measured during perfusion of the renal pelvis with a dilute solution of a radiopaque contrast medium at the rate of $10 \mathrm{ml} / \mathrm{min}$. If there is no obstruction in the collecting system, the differential pressure

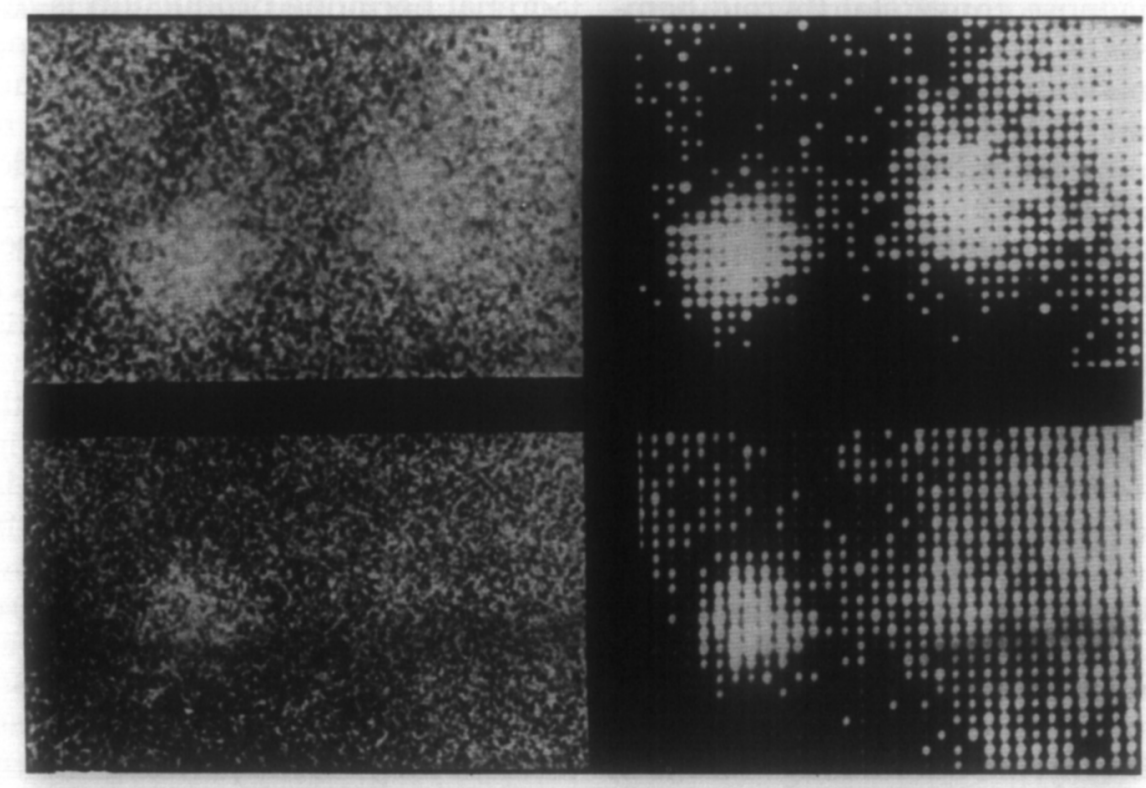

FIG 1.

Radiocholesterol images in a patient with hyperaidosteronism. The top two images are the analog (left) and computer display (right) images obtained in the patient under basal conditions. The adrenals appear symmetrical (in this posterior view the activity in the upper right hand corner of the image is normal hepatic activity). The bottom two images are the corresponding analog and computer views in a repeat study after the patient had undergone dexamethasone suppression. The failure of the left adrenal activity to be suppressed and resultant significant asyrmmetry between the two glands indicate the presence of a left aldosteronoma. (From Thrall $J H_{1}$ Freitas JE, Beierwaltes WH: Adrenal scintigraphy. Semin Nucl Med 1978; 8:23-41. Used with permission.) 


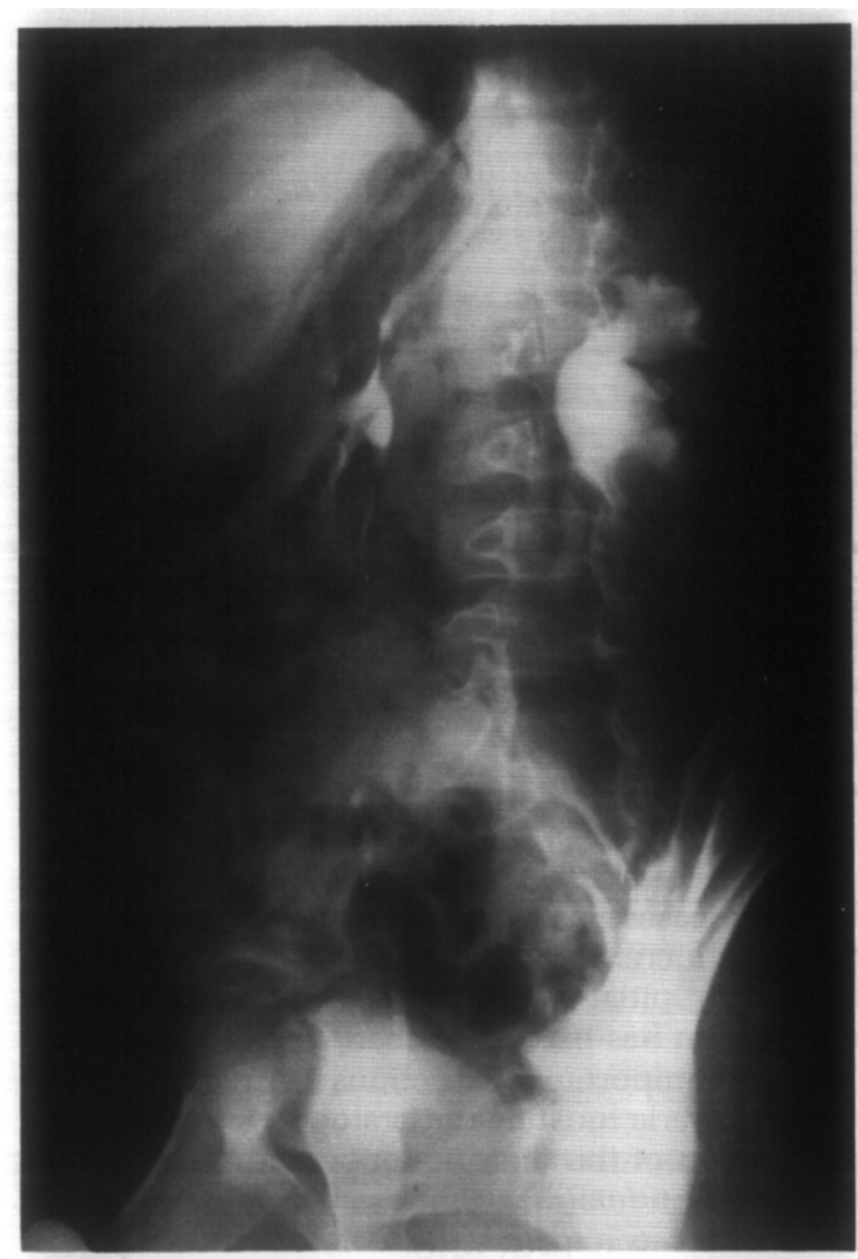

FIG 2.

Excretory urogram in a patient with intermittent left flank pain. The left renal pelvis and calices are mild to moderately dilated. There is the appearance of a narrowing at the left ureteropelvic junction. This case illustrates the usual clinical dilemma: is the collecting system mechanically obstructed, or is this a nonobstructed, dilated system?

between kidney and bladder remains low, less than 10 to $12 \mathrm{~cm} \mathrm{H}_{2} \mathrm{O}$ in normal individuals. In the presence of significant mechanical obstruction, the equilibrium pressure exceeds $15 \mathrm{~cm} \mathrm{H}_{2} \mathrm{O}$. The clinical value of the test has been attested by a number of investigators. ${ }^{26-30}$ The technique is contraindicated in cases of acute pyelonephritis or pyelonephrosis and should be used with caution in patients with bleeding disorders. ${ }^{31}$

In the alternative noninvasive radionuclide technique, a diuretic is used to stimulate urine flow while the rate of clearance of the tracer from the collecting structures is measured. The rationale for the lest comes from the observation that the mean transit time of a tracer through a system is equal to the ratio of the volume of the system divided by the rate of flow through the system (i.e., $\bar{t}=V / F$ ). Thus, in a large dilated collecting system, there is a reservoir effect that results in the prolonged re- tention of a radiotracer or contrast material. Stimulating the flow of urine with a diuretic should reduce the mean transit time of a radionuclide in dilated, but nonobstructed systems by increasing urine flow. On the other hand, in a system with a fixed mechanical obstruction, the system either cannot respond or will respond only minimally to the diuretic, and a failure of tracer washout or perhaps even a further increase in its accumulation in the collecting structures will be observed.

Diuretic augmented urography was first described in the late $1960 \mathrm{~s}^{32}$ In 1978 , O'Reilly et al. described qualitative response patterns for normal collecting systems, dilated nonobstructed systems, and obstructed systems using scintillation probes placed over the kidneys and ${ }^{131}$ I-hippuran as the tracer $^{33,34}$ Koff and coworkers incorporated a gamma camera interfaced to a nuclear medicine computer system and introduced the use of ${ }^{99 \mathrm{~m}} \mathrm{Tc}-$ diethylenetriamine-pentaacetic acid (DTPA) as the tracer. $^{35-37}$ Gamma camera modification of the technique permitted region-of-interest analysis and extended the procedure to evaluation of the ureters as well as the ureteropelvic junction. The use of ${ }^{99 \mathrm{~m}_{\mathrm{H}}}$ Tc-DTPA afforded a significant reduction in the radiation dose and sufficient photon flux for simultaneous imaging and quantitative washout analysis.

The basic procedure protocol involves imaging the kidneys and their collecting structures before and after the administration of a potent, high-ceiling diuretic. In the approach of Koff and coworkers, the patient is studied in a normally hydrated condition. ${ }^{35,37} \mathrm{~A}$ wide-field-of-view gamma camera is used to permit imaging from the level of the kidneys to the bladder, and the patient is imaged while in a sitting position in the posterior view. Infants and small children may be imaged supine or prone. An adult dose of 10 to $15 \mathrm{mCi}$ of ${ }^{99 \mathrm{~m}} \mathrm{Tc}$ DTPA is used. By incorporating a nuclear medicine computer system, an initial flow study is obtained at one frame every 2 seconds to assess relative renal perfusion. After 3 minutes, frames are acquired every 30 to 60 seconds (Fig $3, A$ and $B$ ). The relative uptake in the two kidneys from 1 to $2 \mathrm{~min}$ utes provides a measure of differential glomerular filtration between the kidneys and is a useful adjunctive parameter, particularly in assessing the response to therapy. This analysis requires regions of interest over each kidney and a summation of net activity from the appropriate frames of the flow study (Fig 3,C).

The persistence scope of the gamma camera and/or the computer-acquired images are monitored to determine when the kidneys and collecting structures are completely visualized. When complete visualization has been confirmed, 0.3 to $0.5 \mathrm{mg} / \mathrm{kg}$ of furosemide is administered intrave- 


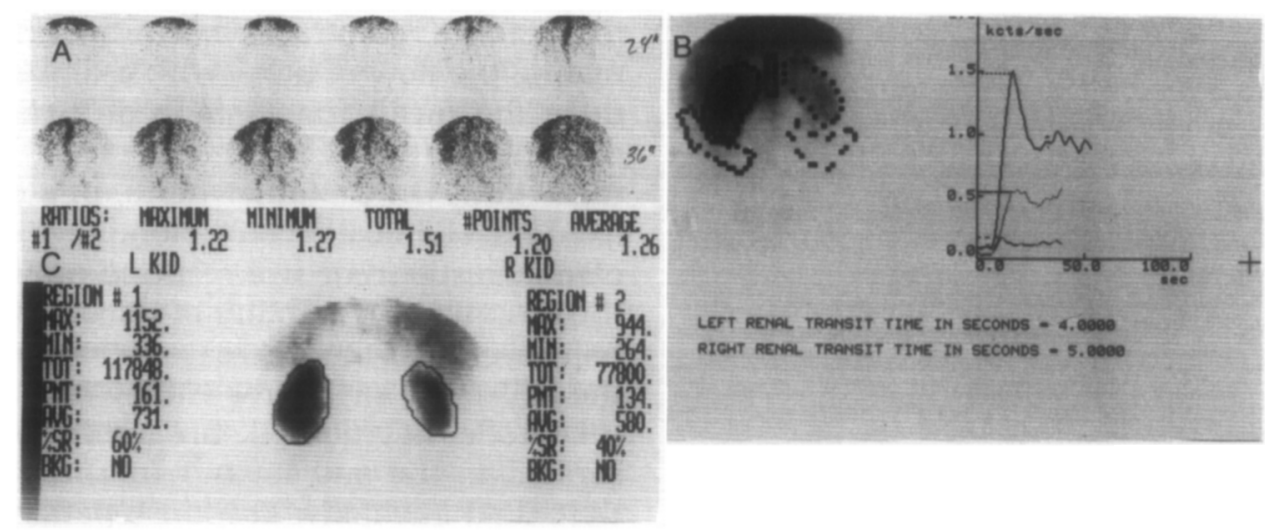

FIG 3.

A, posterior-view flow study obtained by using $15 \mathrm{mCi}{ }^{99 \mathrm{~m} T C-D T P A}$. Each image is for 2 seconds. Note the asymmetry of renal perfusion, with more activity appearing during the arterial phase on the left than on the right. B, regions of interest for the kidneys, aorta, and background areas with corresponding flow study time-activity histograms. The higher peak associated with the left kidney indicates greater perfusion. C, summation of the total counts in the 1 - to 2 -minute interval indicates a $60 \%$ relative contribution from the left kidney and a $40 \%$ relative contribution from the right kidney-a significant difference in the glomerular filtration rate on the two sides. (From Thrall JH, Koff SA, Keyes JW Jr: Diuretic radionuclide urography in the differential diagnosis of hydroureteronephrosis. Semin Nucl Med $1981 ; 11: 89-114$. Used by permission.)

nously. Slow injection over 1 to 2 minutes mmimizes side effects, which are typically infrequent and limited to nausea. Furosemide exerts its diuretic effect by inhibiting sodium and chloride resorption from the proximal and distal tubules and the loop of Henle. It is used because of its rapid onset of action after intravenous administration and its low toxicity. A washout effect can be seen in as little as 30 to 60 seconds. Data collection is continued for a minimum of $\mathbf{1 0}$ minutes and preferably 15 minutes after diuretic injection.

Data analysis involves generating a backgroundcorrected, time-activity histogram for each renal unit, the ureters, and the bladder. The renal regions of interest include the entire kidney and renal pelvis. In normal subjects, the renal time-activity histogram reaches a peak in 3 to 5 minutes; this is followed by a spontaneous washout of activity that is accelerated after the administration of the diuretic. In the normal ureter, there is a flat time-activity curve just above background with a transient spike of activity reflecting the washout from above (Fig 4). ${ }^{34,37}$

In patients with dilated, nonobstructed collecting systems, the time-activity histogram demonstrates a continuous increase in activity or an increase to a broad plateau level and then a variable degree of washout in response to the diuretic (Fig 5). ${ }^{34,37}$ 'The larger the collecting system, the less "crisp" the response. The time-activity curve associated with a dilated, nonobstructed ureter is similar in appearance. ${ }^{37}$

In patients with dilated, obstructed syslems, the time-activity histogram reveals increasing activity or activity that reaches a plateau and then little or no response to the diuretic (Figs 6 and 7,A and B).
In some cases, tracer acciumulation actually continues to increase, and a potentially confusing pattern of an initial slight decrease followed by reaccumulation has been described.

Several important restrictions of the aforementioned criteria must be understood for appropriate application of the diuretic renogram. Most important, the rationale for the test is not met if renal function is so impaired that there is no response to the diuretic (Fig 8). ${ }^{37}$ If the creatinine level is above $5.0 \mathrm{mg} / \mathrm{dL}$, the kidney is poorly visualized, or the collecting systems are not visualized, the aforementioned criteria for distinguishing obstructed from nonobstructed systems clearly do not apply. The ability to change the flow rate is so greatly impaired under these conditions that a washout phenomenon would not be observed even in an unobștructed system. Moreover, an "intermediate" response must be interpreted with caution. With good renal function and a truly unobstructed system, a washout of $\mathbf{5 0 \%}$ or greater is typically seen within 10 minutes of diuretic injection. Patients with "intermediate" or "indeterminant" responses merit close follow-up with the further proviso that the diuresis renogram is simply one evaluative method. The test does not provide a definitive answer in every case. Systems can also convert from one pattern to another if an infectious process is part of the clinical problem.

In addition to the initial diagnosis of urinary tract obstruction, the diuresis renogram is valuable in assessing the results of surgery (Fig 9). Residual urinary tract dilatiop is extremely common, even after successful pyeloplasty or ureteral neocystostomy. The diuresis renogram provides an objective means of demonstrating that the collecting system 

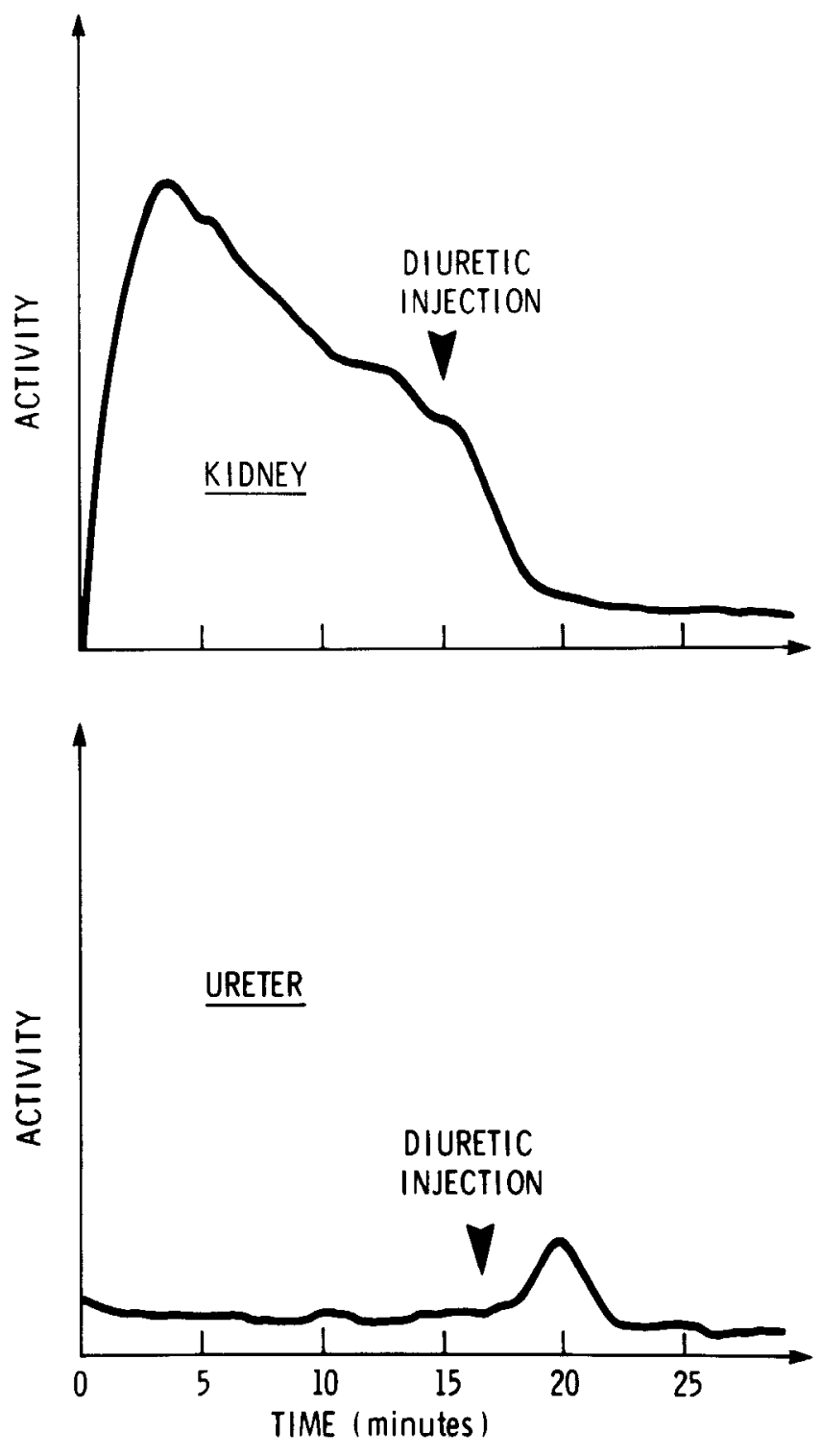

FIG 4.

Normal patterns for the renal and ureteral time-activity histograms in nonobstructed and nondilated systems. Note the timing relationship between the accelerated washout from the kidney and the spike of ureteral activity. (From Thrall JH. Koff SA, Keyes JW Jr: Diuretic radionuclide urography in the differential diagnosis of hydroureteronephrosis. Semin NuCl Med 1981; 11:89-114. Used by permission.)

is unobstructed ${ }^{38}$ Renal transplantation also involves a high incidence of collecting system dilation, and the diuresis renogram is useful in this setting as well. ${ }^{39}$

\section{RADIONUCLIDE CYSTOGRAPHY}

Radionuclide methods have become increasingly accepted in the evaluation of vesicoureteral reflux, most importantly in children with urinary
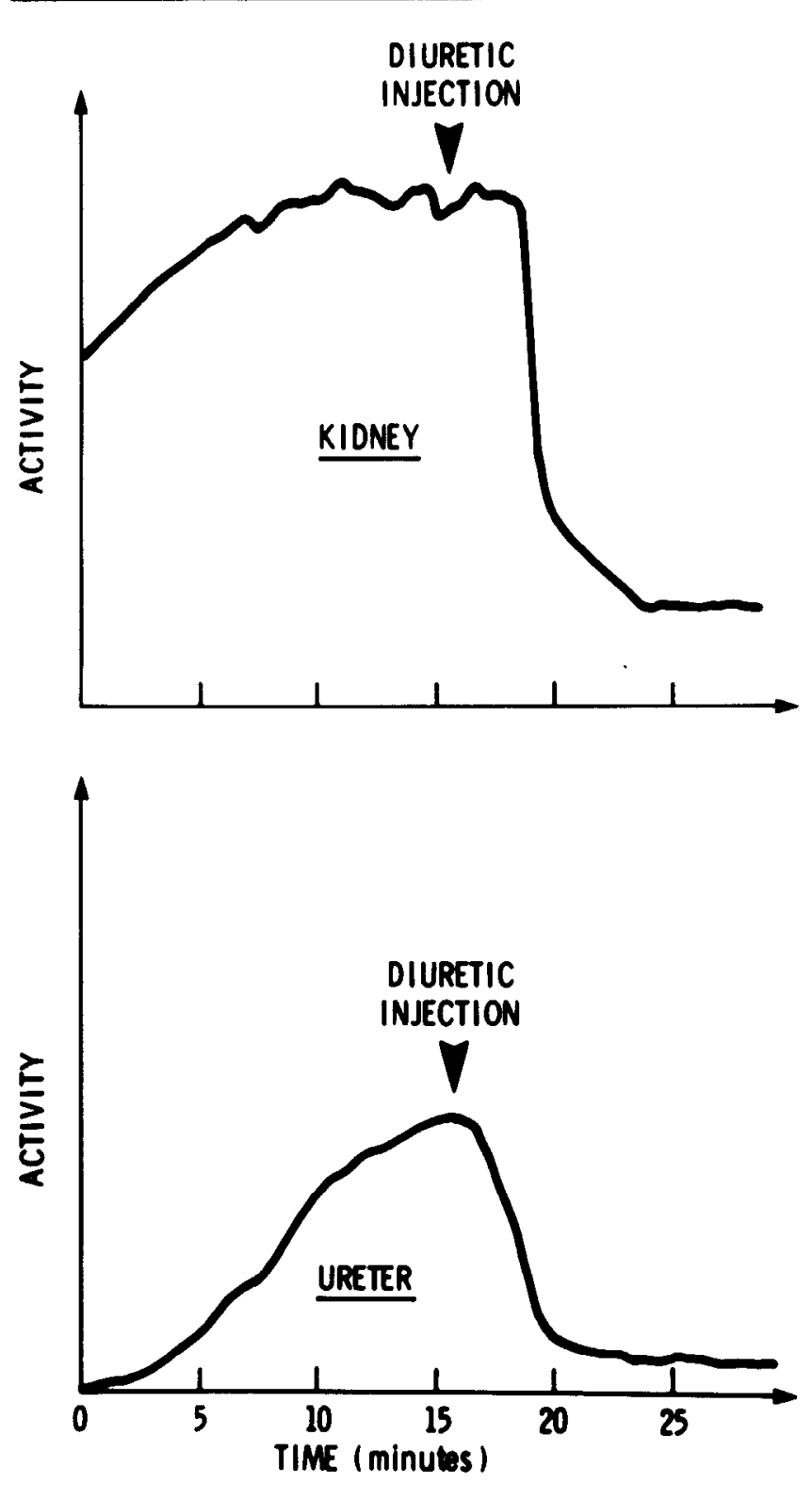

\section{FIG 5.}

Time-activity histograms for dilated, nonobstructed kidneys and ureters. The diuretic response is dramatic and unequivocal in the illustrated curve. Lesser degrees of washout response are frequently seen in mairkedly dilated kidneys and kidneys with diminished function. (From Thrall JH, Koff SA, Keyes JW Jr: Diuretic radionuclide urography in the differential diagnosis of hydroureteronephrosis. Semin Nucl Med 1981; 11:89-114. Used by permission.)

tract infections. Unremitting reflux may be associated with damage to the kidney and may, in later life, lead to hypertension and even chronic renal failure. In patients adequately treated with antibiotics, vesicoureteral reflux typically resolves with timc and does not require surgery in most cases.

The traditional method for following children with reflux has been the voiding cystourethrogram. However, this radiographic procedure is as- 

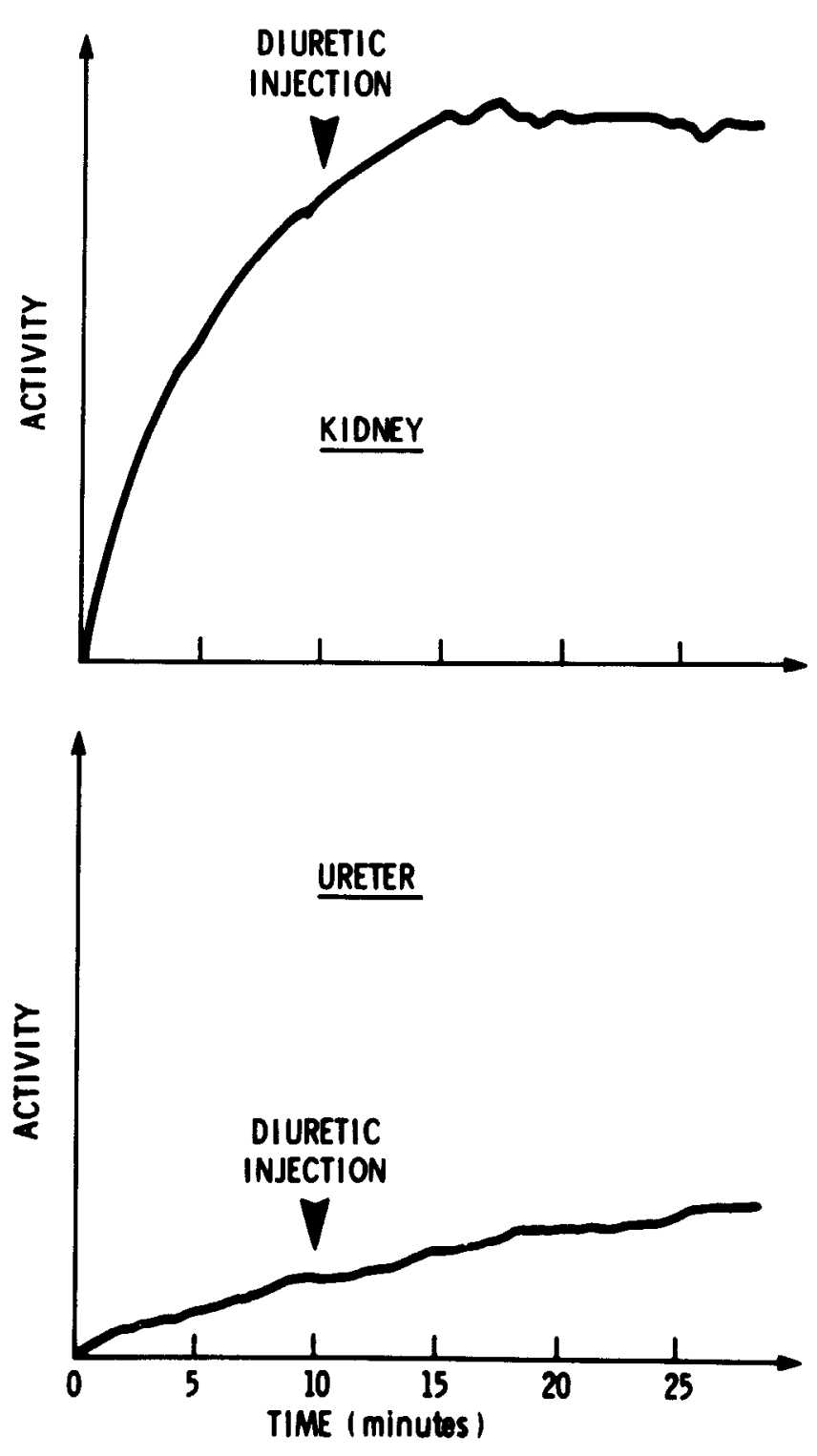

FIG 6.

Renal and ureteral time-activity patterns for obstructed systems The renal activity fails to wash out or diminish following diuretic injection. (From Thrall JH, Koff SA, Keyes JW Jr: Diuretic radionuclide urography in the differential diagnosis of hydroureteronephrosis. Semin Nucl Med 1981; 11:89-114. Used by permission.)

sociated with a significantly higher radiation dose to the gonads than is the nuclear medicine technique and is, for a number of reasons, less sensitive in detecting reflux. The radionuclide study permits continuous monitoring during the filling and micturition phases. ${ }^{40-42}$ Moreover, the high contrast resolution of the radionuclide technique permits the detection of reflux volumes on the order of $1.0 \mathrm{ml}$. A frequently employed strategy is to obtain an initial radiographic study for detailed anatomic evaluation, with follow-up studies performed scintigraphically to minimize the radiation dose to the gonads and to take advantage of the high sensitivity of the radionuclide test.

The radionuclide cystogram is typically performed as a three-phase procedure: continuous monitoring during filling of the bladder, micturition, and a postvoiding period. The procedure may be done simply to determine the presence or absence of reflux or to measure postvoiding residue in the bladder. The radionuclide cystogram may be modified to record pressure-volume relationships in the bladder for correlation with time of reflux.

A gamma scintillation camera interfaced to a nuclear medicine computer system is used for the procedure. With the patient positioned so that the bladder, ureters, and kidneys are in the field of view, the study is obtained as a dynamic sequence at the rate of one frame every 5 or 10 seconds. Analog images may also be obtained every 30 to 60 seconds to integrate activity during different phases of the study. ${ }^{99 \mathrm{~m}} \mathrm{Tc}$ as sodium pertechnetate or another nonabsorbable form is used as the radiotracer. A solution of $1 \mathrm{mCi} / 500 \mathrm{ml}$ provides a sufficient concentration.

Catheterization of a child is an important step in the procedure, and all personnel involved should be taught the appropriate sterile technique. The patient's cooperation is extremely valuable to avoid premature voiding around the catheter and resultant contamination of the imaging field. The bladder should be filled slowly. As capacity is reached, voiding may occur spontaneously, especially in younger children and infants.

In a normal study, no tracer is demonstrated in the region of the ureters or kidneys (Fig 10). Reflux is readily detected by the presence of activity above the bladder and may be graded (Fig 11). Willi and Treves have suggested that the detailed grading system used radiographically is not feasible for radionuclide imaging because of the more limited spatial resolution. ${ }^{42}$ However, it is still possible to grade the degree of reflux as minimal (reflux confined to the ureter), mild to moderate (reflux reaches the pelvicaliceal system), or severe (distended collecting system and/or redundant ureter).

As noted earlier, one of the advantages of the radionuclide technique is continuous monitoring. In a small percentage of patients, reflux is demonstrated only during the filling phase. In the majority $(\mathbf{8 0} \%)$, reflux is seen during both the filling and micturition phase, and in the remainder, it is seen only during voiding. ${ }^{41,42}$ Although it requires catheterization of the bladder, the method of direct cystography is felt to be more accurate than is indirect cystography. ${ }^{43}$

The volume of the bladder and the residual vol- 


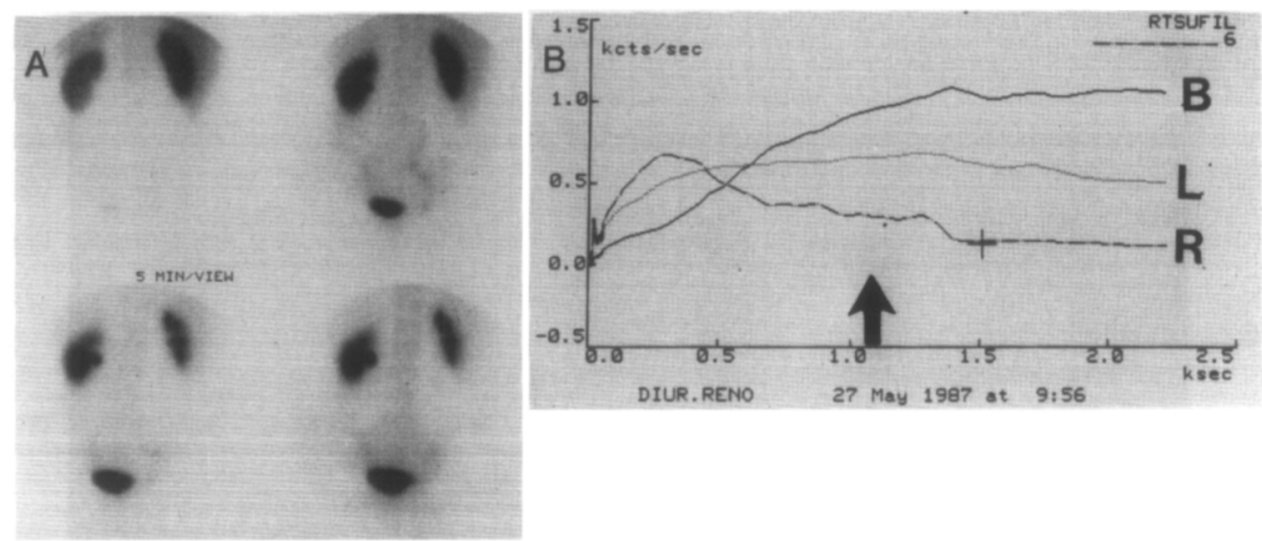

FIG 7.

A, selected images obtained from the posterior view during a diuresis renogram. Note the delayed visualization of the left renal collecting structures. There is some degree of dilation bilaterally. The tracer was injected at approximately 17 minutes into the study (between the time of the second image on the top row and the first image on the bottom row). The collecting structures on the right respond promptly to the diuretic. On the left, there is progressive pooling, particularly in the lower poles. B, time-activity histograms for the bladder $(B)$ and the left $(L)$ and right $(R)$ kidneys. The vertical arrow indicates the time of furosemide (Lasix) administration. The right kidney demonstrates a broad, somewhat delayed peak compatible with a degree of dilation. There is some spontaneous washout that is further accelerated after administration of the diuretic. The left kidney fails to respond to the diuretic, which indicates a significant mechanical obstruction.

ume after voiding can be readily calculated by measuring the change in count rate before and after voiding and relating the change to the urine volume. This approach assumes a constant attenuation, an assumption that is probably slightly incorrect, but reasonable. ${ }^{42}$

\section{CAPIOPRIL RENOGRAPHY}

A third potential renal interventional study has been proposed for the evaluation of patients with suspected renal vascular hypertension. Captopril is an angiotensin-converting enzyme inhibitor that has been used for the treatment of hypertension. Diminished renal function and even renal failure have been described in patients receiving captopril therapcutically, and captopril is linked to the presence of significant renal vascular hypertension. ${ }^{44,45}$ The postulated mechanism is a reduction in the tone of the postglomerular arterial bed as normally mediated hy angiotensin II. This process results in a decrease in the glomerular filtration rate in pa-

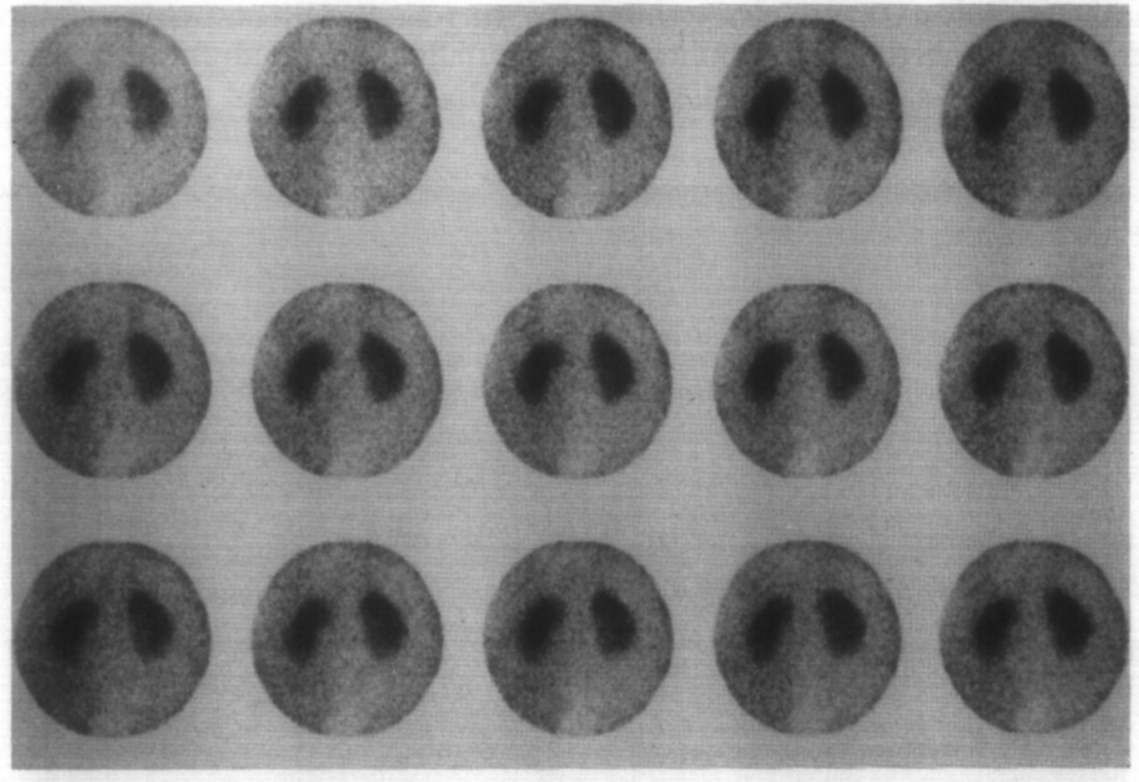

FIG 8.

Sequential images in a patient with acute renal failure. Note the failure of the collecting systems to be discretely visualized. There is prolonged, rather static retention of tracer bilaterally. In this setting, the diuresis technique is inappropriate. 


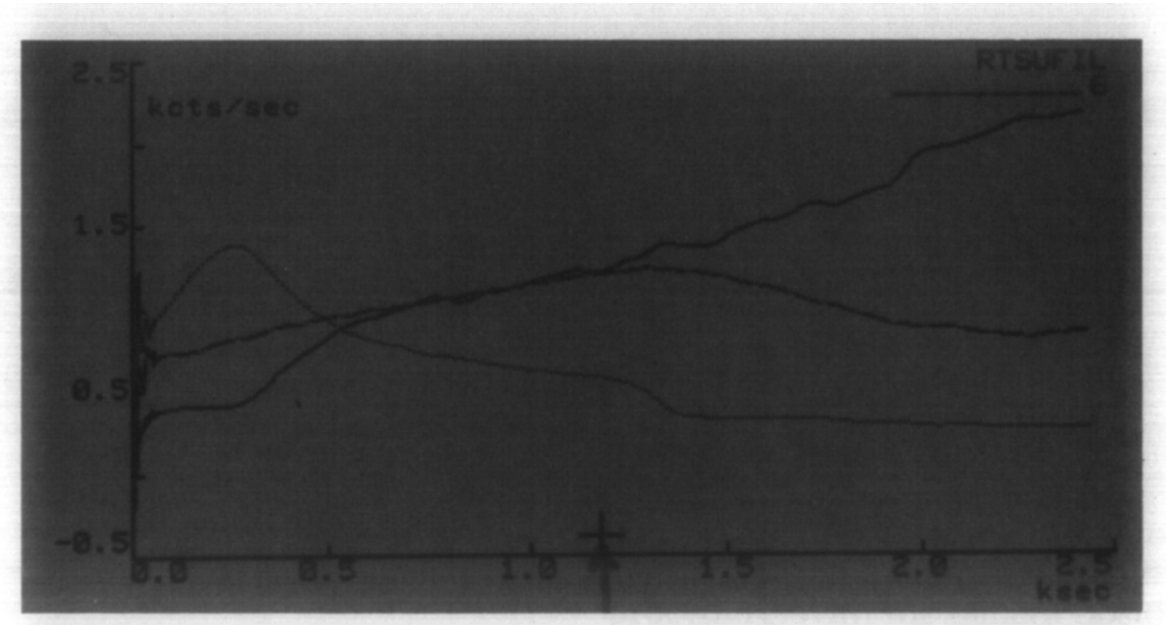

FIG 9.

Follow-up time-activity histograms from the patient illustrated in Figure 7. Following a left pyeloplasty, there is some response to the diuretic, which indicates some relief of the obstruction. Left kidney, middle curve; right kidney, dotted curve; bladder, upper continuous curve.

tients with significant obstructions of the renal artery.

Several investigators recognized that the undesirable effects of captopril on renal function actually suggested the possibility of a diagnostic intervention to enhance the detection of renal vascular disease. ${ }^{4-48}$ In brief, a renogram is performed before and after the administration of captopril. In normal subjects and hypertensive patients without renal vascular disease, captopril does not significantly affect the renogram. In patients with a significant renal vascular abnormality, the renogram is altered, and diminished glomerular filtration and diminished effective renal plasma flow are demonstrated. The accuracy of plasma renin measurements and renal vein renin determinations in evaluating hypertensive patients has also been shown to be improved when using cap- topril. ${ }^{49}$ Early experience in experimental models has been very encouraging. Whether this experience can be translated from the acute experimental model to patients with chronic long-standing disease remains to be established, although initial clinical results have becn promising. ${ }^{50}$ However, this is another elegant example of how, for a particular diagnostic purpose, the ability to intervene and change a complex physiological system can improve the utility of a procedure that had essentially been abandoned.

\section{CARDIAC INTERVENTIONS}

In many subjects with significant and even potentially life-threatening coronary artery disease, cardiac function is normal under basal or resting

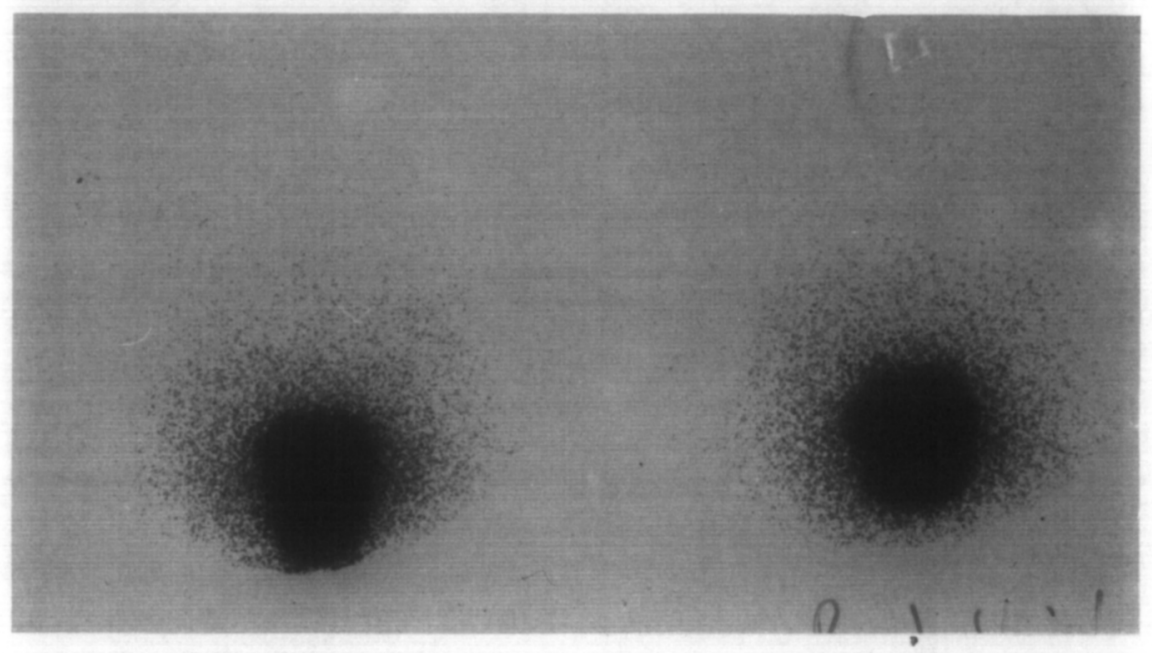

FIG 10.

Nomal direcl radionuclide cystogram. No tracer is seen above the level of the bladder. Apparent perivesicle background activity is due to scatter from the photons arising in the bladder. 


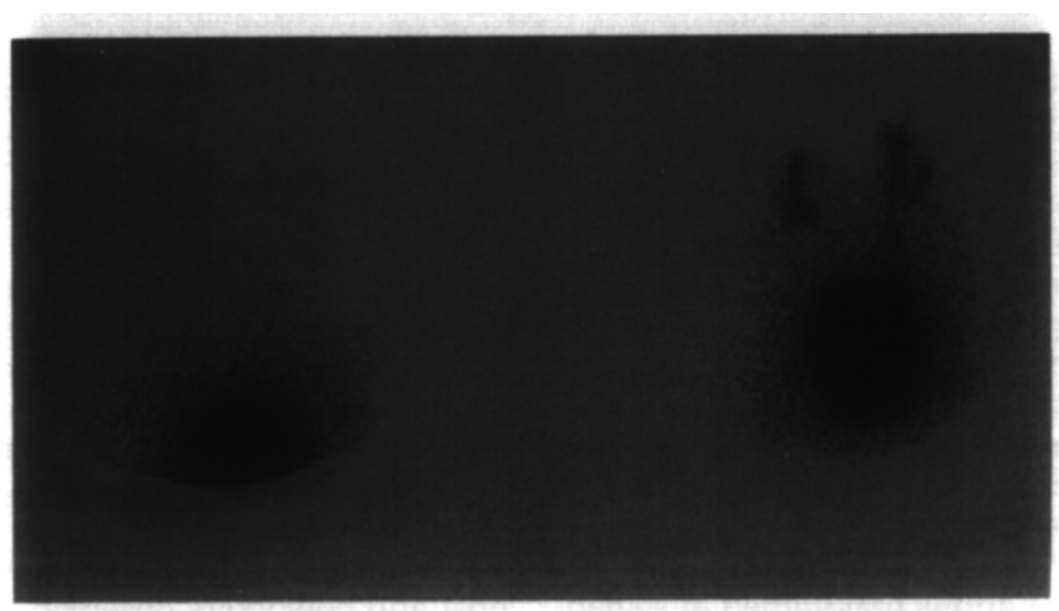

FIG 11.

Abnormal direct radionuclide cystogram. The image on the left was obtained during filling and the figure on the right during voiding. No reflux is demonstrated during the filling phase. During the voiding phase, there is significant reflux into the intrarenal collecting structures on both sides, which are mildly dilated.

conditions, and underlying abnormalities in the coronary circulation only become manifested under conditions of exercise or other stress. Clinically, it has been recognized for decades that patients with angina pectoris experience their pain in response to physical work, exposure to cold, and even emotional upset. These "interventions" of normal daily living form the basis for enumerable forms of cardiac stress testing and interventions aimed at detecting occult coronary disease and measuring cardiac reserve responses.

In the discussion of cardiac stress testing, it is often puzzling to newcomers that significant stenoses of the coronary arteries have so little apparent effect on coronary circulation at rest. An understanding of the relationship between flow reserve and degree of vessel stenosis is important in developing this understanding. In brief, the resting coronary blood flow may be augmented as much as 5- to 6-fold in response to exercise. Thus, even a vessel with significant stenosis may still be adequate under basal conditions. However, exercise or other stress increases oxygen demands and, therefore, flow demands. The fixed stenosis then causes diminished flow reserve, and ischemia may develop in the vascular distribution distal to the narrowing. Thus, the goal of exercise/stress testing in cardiac interventional analysis is the same whether the measuring technique is an electrocardiogram (ECG), a ${ }^{201} \mathrm{Tl}$ myocardial perfusion scintigram, or a radionuclide ventriculogram. Only the marker of ischemia is different. For the ECG, characteristic changes, most notably ST segment depression, are detected in ischemic areas. Electrolyte flux across the ischemic cell membranes is impaired and causes the characteristic changes seen electrocardiographically. With the ${ }^{201} \mathrm{Tl}$ myocardial perfusion scan, regional ischemia is manifested as a relative decrease in blood flow and therefore in tracer delivery as well as a diminished ability of the ischemic myocardial cells to accumulate the tracer. The characteristic appearance on the perfusion scan in ischemic areas is a lower relative tracer localization than in nonischemic areas. For the radionuclide ventriculogram, ischemia is manifested by abnormalities in regional wall motion and associated impairment of quantitative measures of ventricular function, most importantly the ejection fraction. From the foregoing, it should be apparent that, whichever marker of ischemia is used, it is vital to the success of the procedure that sufficient stress be applied to genuinely challenge the coronary flow reserve. A number of paraneters have been used to assess the adequacy of stress, including the response of the heart rate, the blood pressure, and combinations of the two.

\section{THALLIUM 201 MYOCARDLAL PERFUSION IMAGING}

In the early experience with radionuclide techniques for cardiac intervention studies, there was a spirited debate over the relative merits of ${ }^{201} \mathrm{Tl}$ perfusion imaging and exercise radionuclide ventriculography. Clinical results with both techniques appeared reasonable, but in the last half of the $1980 \mathrm{~s}$ the stress thallium scan has become the clear-cut procedure of choice in most medical centers. There are several reasons for this preference. These include the ease with which the stress thallium scan can be "grafted" onto a standard treadmill stress ECG evaluation and the relative technical simplicity of the procedure. The exercise radionuclide ventriculogram approach requires specialized equipment such as ECG gating devices to control data acquisition and requires significant 
training of personnel in analysis of the ventriculographic data to obtain reproducible results. The exercise radionuclide ventriculogram remains important in specific circumstances but is clearly not used as frequently as ${ }^{201} \mathrm{Tl}$ imaging in the diagnosis of coronary artery disease.

Although there are various techniques for ${ }^{201} \mathrm{Tl}$ imaging, the basic principles are now well established. 'The procedure is most frequently performed in conjunction with a formal treadmill ECG. Standard exercise protocols are used, and most laboratories adapt the rigorousness of the protocol to the patient's clinical condition. For injection of the ${ }^{201} \mathrm{Tl}$, an intravenous line is started beforc exercise begins and is maintained at a keepopen level. When the physician exercising the patient feels that the point of maximum stress is being approached or has been achieved, the thallium is injected as an intravenous bolus in a dose of 2 to $3 \mathrm{mCi}$. Exercise is continued for 1 to $2 \mathrm{~min}$ utes if possible to ensure that the initial uptake of the tracer reflects the perfusion pattern at peak exercise as closely as possible. The thallium is rapidly cleared from the blood with an $85 \%$ extraction efficiency in the myocardium. Its regional distribution correlates well with regional perfusion except at extremes of high and low flow. ${ }^{51}$

In the early experience with ${ }^{201} \mathrm{Tl}$ stress myocardial perfusion imaging, there was considerable debate regarding the ideal time for beginning the imaging procedure. Some investigators advocated waiting as long as 15 or 20 minutes to allow the complete blood clearance of ${ }^{201} \mathrm{Tl}$ and its uptake in the myocardium. It has now been established that imaging should begin as soon as possible following the stress and that a delay of 20 minutes may actually reduce the sensitivity of the procedure because of early redistribution and masking of some exercise-induced abnormalities. ${ }^{52,53}$

For the imaging portion of the procedure, either planar or tomographic techniques continue to be acceptable. The key to success is probably the adoption of a standard imaging protocol and its meticulous application. For planar imaging, protocols based on a fixed time per image are preferred over protocols based on counts per image. This procedure permits a direct comparison of regional uptake in the myocardium between early and delayed imaging and eliminates the problem of changing activity levels in the background, which can be confusing in count-based protocols. Under the time approach, images are obtained for 8 to 10 minutes per view. Some authors reconmend beginning with the left anterior oblique view because they feel that it provides the greatest diagnostic information. A minimum of three views is obtained. For tomographic studies employing single-photon emission computed tomography
(SPECT), the merits of 180 - vs. 360-degree acquisition arcs are still being debated ${ }^{54,55}$ The weight of evidence appears to be shifting in favor of the 180degree-arc approach. ${ }^{55}$ The arc chosen is from a 45-degree right anterior oblique to a 135-degree left posterior oblique position. This arc minimizes soft-tissue attenuation between the heart and the detector. To minimize the redistribution phenomenon, data acquisition for tomography should ideally be kept under 20 to 25 minutes.

In the initial experience with ${ }^{201} 11$ myocardial perfusion imaging, patients received two separate injections of the radiotracer to assess the basal uptake pattern and the pattern at peak exercise. Pohost and coworkers observed that thallium undergoes a continuous redistribution process after injection ${ }^{56}$ that permits reimaging after a time delay to assess whether defects noted at the time of initial imaging persist or fill in. Delayed images are typically obtained at 2 to 4 hours. It is important to standardize the time of delay to provide reproducible and comparable results between patients.

The patterns of initial and delayed uptake form the basis of the diagnostic scheme for stress ${ }^{201} \mathrm{Tl}$ imaging (Table 2). In normal subjects and patients without coronary artery disease, the immediate postexercise study demonstrates a uniform uptake in the myocardium in all views (Fig 12). The uptake diminishes but is still uniform at the time of delayed imaging. Several investigators have developed quantitative criteria for the percentage of ${ }^{201}$ Tl that should normally "washout" between early and delayed imaging. ${ }^{53,57,58}$ The level of exercise achieved, the length of delay, intercurrent activity, and even the sex of the patient all influence these measurements, which makes it difficult to provide unqualified quantitative criteria. As a broad rule of thumb in subjects achieving good exercise levels, a decrease in activity on the order of $25 \%$ to $40 \%$ should be seen at 2 hours.

On ${ }^{201} \mathrm{Tl}$ scintigrams the hallmark of exercise-induced ischemia as a marker of coronary artery disease is a defect on the postexercise study that "normalizes" or becomes less apparent on delayed images (Table 2 and Figs 13 and 14,A and B). The lower relative flow to the ischemic segment under stress conditions results in less tracer delivery and uptake. During the several hours of the recirculation phase, the lower gradient between the ischemic cells and the circulating levels of ${ }^{201} \mathrm{Tl}$ as compared with normal areas results in less washout and, in some cases, an actual net increase in tracer activity, which accounts for the normalization phenomenon. Scarred myocardium from a prior infarction does, not accumulate tracer during the recirculation phase and remains photopenic on both immediate and delayed images (Fig 15, A and $B$ ). 
TABLE 2.

\begin{tabular}{|c|c|c|}
\hline $\begin{array}{l}\text { Postexercise } \\
\text { Pattern }\end{array}$ & Delayed Pattern & Interpretation \\
\hline Normal & Normal & $\begin{array}{l}\text { Normal; no significant coronary } \\
\text { artery disease }\end{array}$ \\
\hline Abnormal & Normal & $\begin{array}{l}\text { Transient ischemia; significant } \\
\text { coronary artery disease }\end{array}$ \\
\hline Abnormal & Abnormal (no change) & $\begin{array}{l}\text { Prior infarction with scar; significant } \\
\text { coronary artery disease but no } \\
\text { evidence of ischemia }\end{array}$ \\
\hline Abnormal & $\begin{array}{l}\text { Partial segmental } \\
\text { normalization }\end{array}$ & $\begin{array}{l}\text { Transient ischemia; significant } \\
\text { coronary artery disease with } \\
\text { possible coexisting prior } \\
\text { myocardial infarction and scar }\end{array}$ \\
\hline Normal & Abnormal & $\begin{array}{l}\text { "Reverse redistribution": intermediate } \\
\text { probability of coronary artery } \\
\text { disease }\end{array}$ \\
\hline
\end{tabular}

A pattern of "reverse" redistribution has been recognized that consists of an apparent relative worsening of a stress-induced defect or the development of a new defect on delayed images in a previously normal area. ${ }^{59,60}$ The significance and mechanism of this pattern have been studied without conclusive results. Silberstein and DeVries observed the pattern in $5 \%$ of a series of 785 patients. ${ }^{\text {bo }}$ Eleven of 20 patients undergoing coronary arteriography had significant coronary stenoses $1>50 \%$ luminal diameter narrowing). The predictive value of the sign was only $55 \%$, a lower figure than has been reported in some earlier papers. ${ }^{59}$ The proposed mechanisms are all essentially based on the hypothesis that normal and ischemic myocardia have different washout rates. In clinical practice, it is prudent to record the presence of the reverse-redistribution phenomenon while recognizing that, by itself, it indicates only an intermediate likelihood of coronary artery disease and may be present in either ischemic or normally perfused areas.

The interpretation of ${ }^{201} \mathrm{Tl}$ scintigrams is challenging, and a number of pitfalls need to be rec-
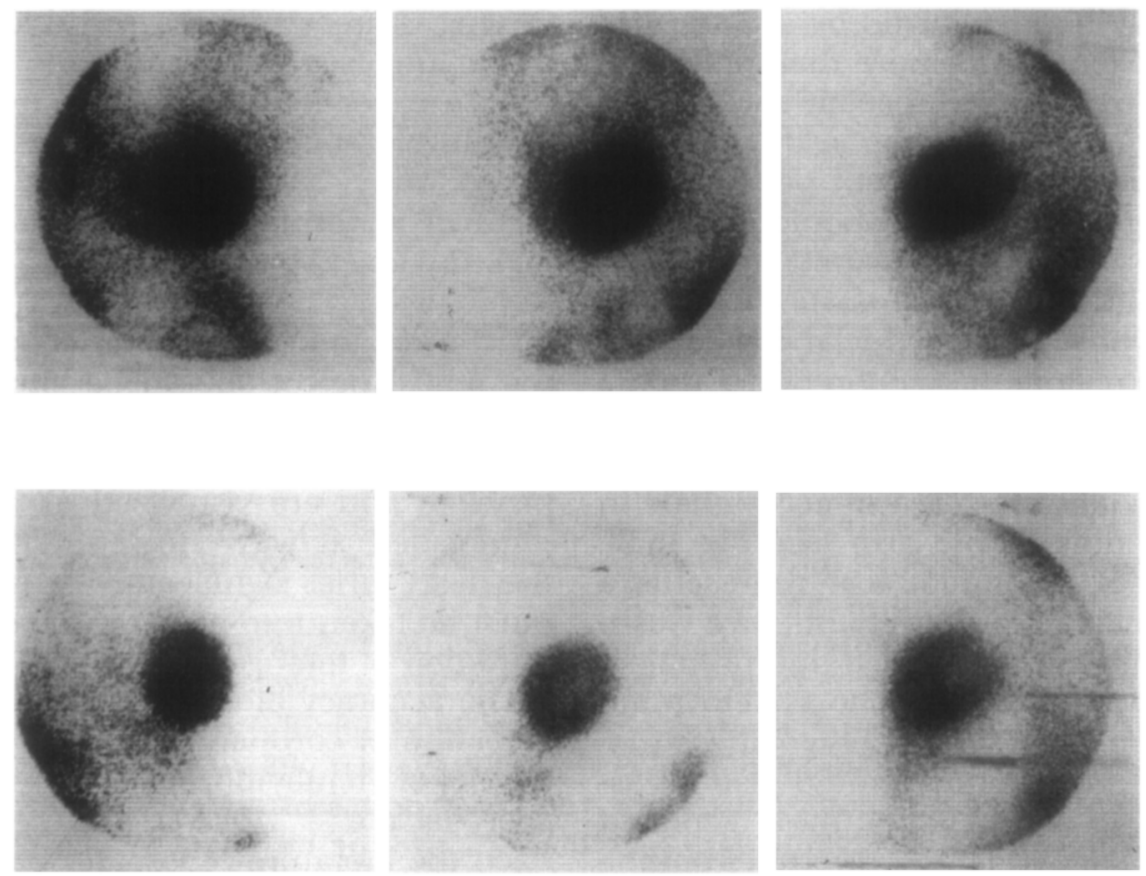

FIG 12.

Immediate postexercise (top row) and delayed images (bottom row) from a patient with no significant coronary artery disease. The initial uptake is uniform with a good target-to-background ratio, and there is uniform activity at the time of delayed imaging (anterior 45-degree left anterior oblique and 70-degree left anterior oblique images from left to right). 

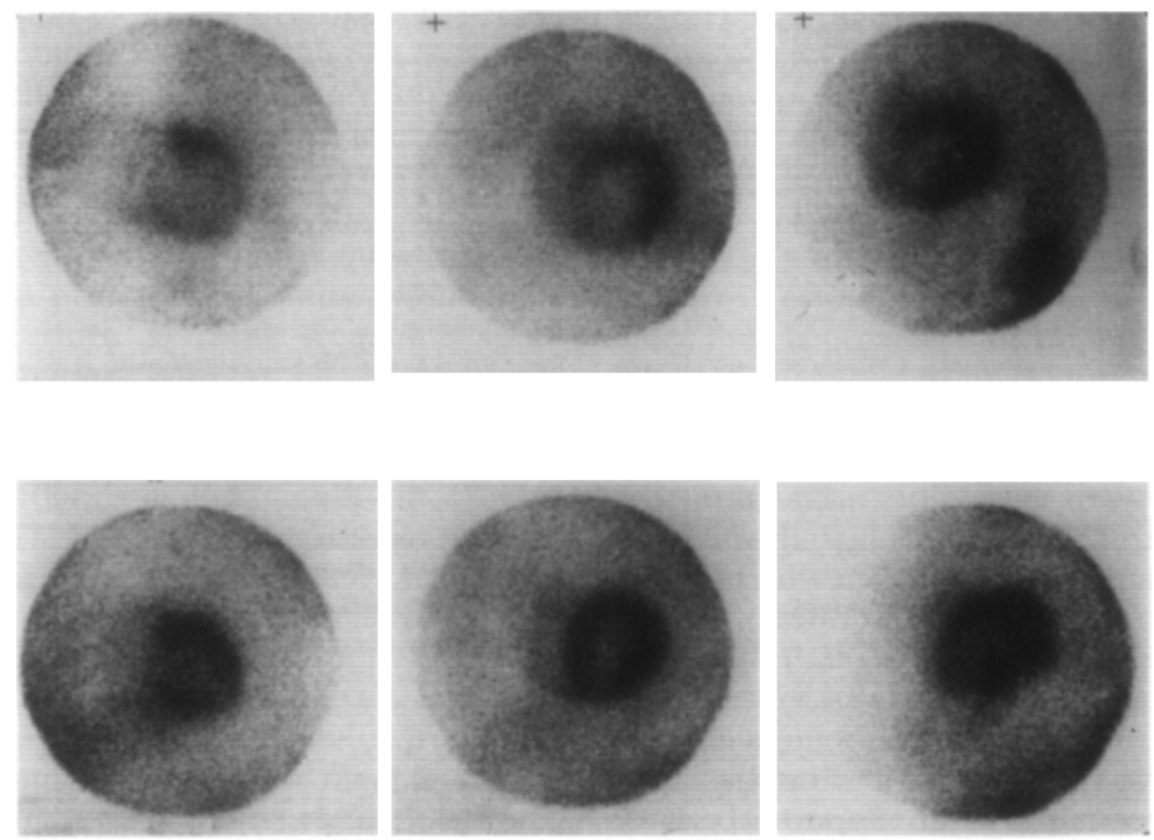

FIG 13.

Immediate postexercise (top row) and 2-hour delayed images (bottom row) in a patient with $>90 \%$ stenosis of the left anterior descending coronary artery. Note the large defect in the septum and anterior wall that essentially completely fills in or normalizes at the time of delayed imaging. This is the classic pattern of exercise-induced ischemia. The myocardium is still viable, but the reduced blood flow during peak exercise results in a relative defect.

ognized. Some degree of inhomogeneity may result from background activity and attenuation artifacts. In women, attenuation due to an overlying breast can cause spurious defects, and some clinics use a breast binder to minimize the effect. The breast artifact can usually be recognized by a pattern of attenuation that often affects more than one myocardial region as well as the adjacent background areas. The left hemidiaphragm can also cause spurious defects, depending on patient position during imaging. The gamma camera should be moved to obtain the lateral view while the patient remains supine. The potential for diaphragmatic attenuation artifact can be further reduced by using a steep left anterior oblique projection rather than a true left lateral. Some investigators have advocated a sitting position.

Occasionally, significant ${ }^{201} \mathrm{Tl}$ will accumulate in the venous structures of the arm (see Fig 15,B). This distribution of the radiotracer represents a reservoir that can actually alter quantitative washout parameters and confuse image interpretation. Injection into the medial or basilic system minimizes the problem. The subdiaphragmatic structures and general background should also be assessed in all cases. If patients have achieved maximum exercise, blood flow is diverted from the splanchnic bed, and there will be minimal or no activity visualized in the liver. With minimal or inadequate exercise, hepatic activity is frequently intense.
The amount of lung background has become recognized as an important secondary diagnostic parameter ${ }^{61,62}$ Patients experiencing impaired ventricular function together with increased pulmonary venous pressure during exercise demonstrate varying degrees of increased thallium localization in the lung (see Figures 15,A and 14,A and B). Lung uptake exceeding $50 \%$ of the myocardial level is definitively abnormal and, in some cases of "balanced" three-vessel disease, may be the only indicator of abnormality. Variable degrees of physiological apical thinning are seen on ${ }^{201} \mathrm{Tl}$ scintigraphy. Each observer must develop a feeling for the range of normal variation, but in general, the apical defert should be small and represent only a few degrees of the circumference. The valve planes of the heart are usually not a diagnostic problem and are visualized as areas of normally diminished activity. However, the orientation of the heart varies significantly between subjects and must be taken into account, especially in the presentation of tomographic data.

The accuracy of stress ${ }^{201} \mathrm{Tl}$ scintigraphy for the detection of coronary artery disease has been the subject of literally dozens of investigations over the past decade. ${ }^{63-72}$ Okada and coworkers summarized the literature reports for planar thallium imaging and visual nonquantitative interpretation of images. $^{72}$ In 1,817 patient studies from 24 series, exercise ${ }^{201} \mathrm{Tl}$ studies had a sensitivity of $82 \%$ and a specificity of $91 \%$ in detecting coronary artery 


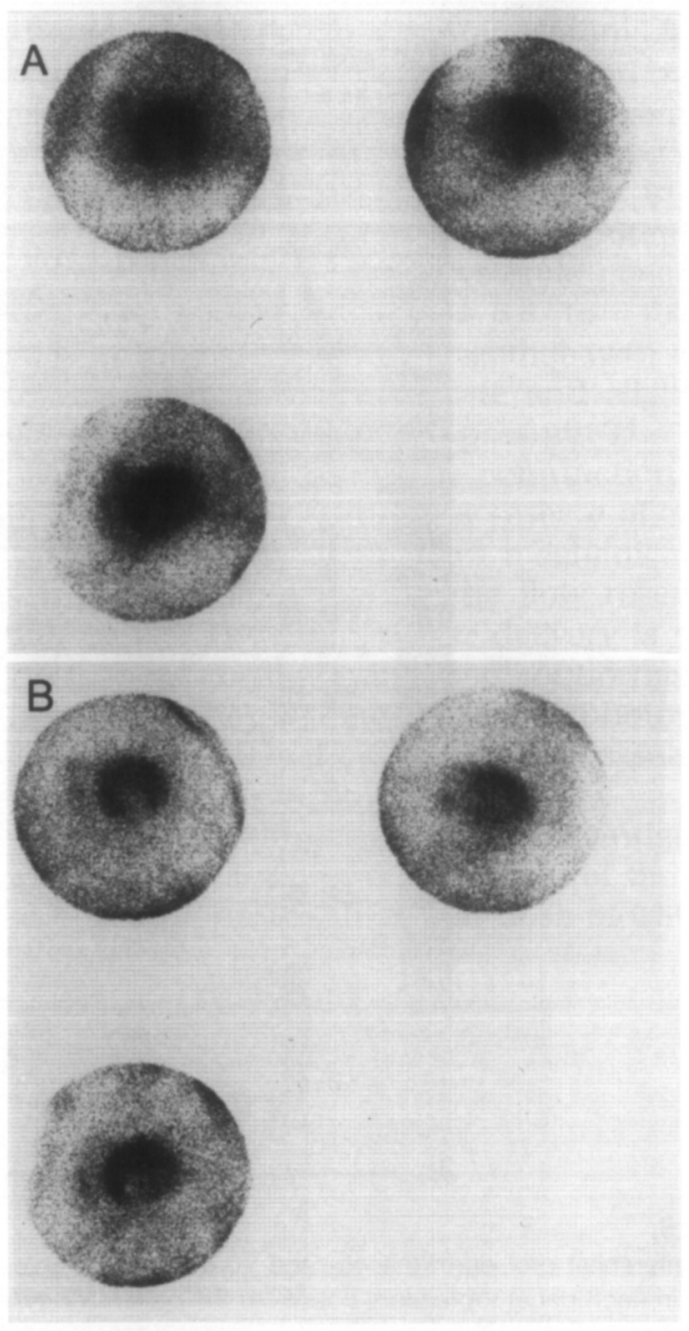

FIG 14.

A, immediate postexercise images in a patient with a significant left anterior descending lesion. There is diminished relative activity in the septal and apical areas. There is increased lung background. The lack of activity below the heart in the structures of the splanchnic bed indicates that a good level of exercise was achieved. B, two-hour delayed images reveal an essentially complete fill-in or normalization. The clearance of the lung background activity and the increase of activity in the liver are best seen by comparison of the respective 45-degree left anterior oblique views (upper left)

disease. Exercise ECG results in the same patient population yielded a sensitivity of $60 \%$ and a specificity of $81 \%$.

More recently, quantitative and tomographic methods have been applied in an attempt to improve study accuracy and reproducibility of interpretation..$^{53-55,73-82}$ The most widely used quantitalive method involves the creation of a circumferential profile of thallium uptake in the myocardium for postexercise and delayed images, ${ }^{77,78}$ with a calculation of the washout percentages and a comparison to "standard" curves from populations of normal subjects or subjects

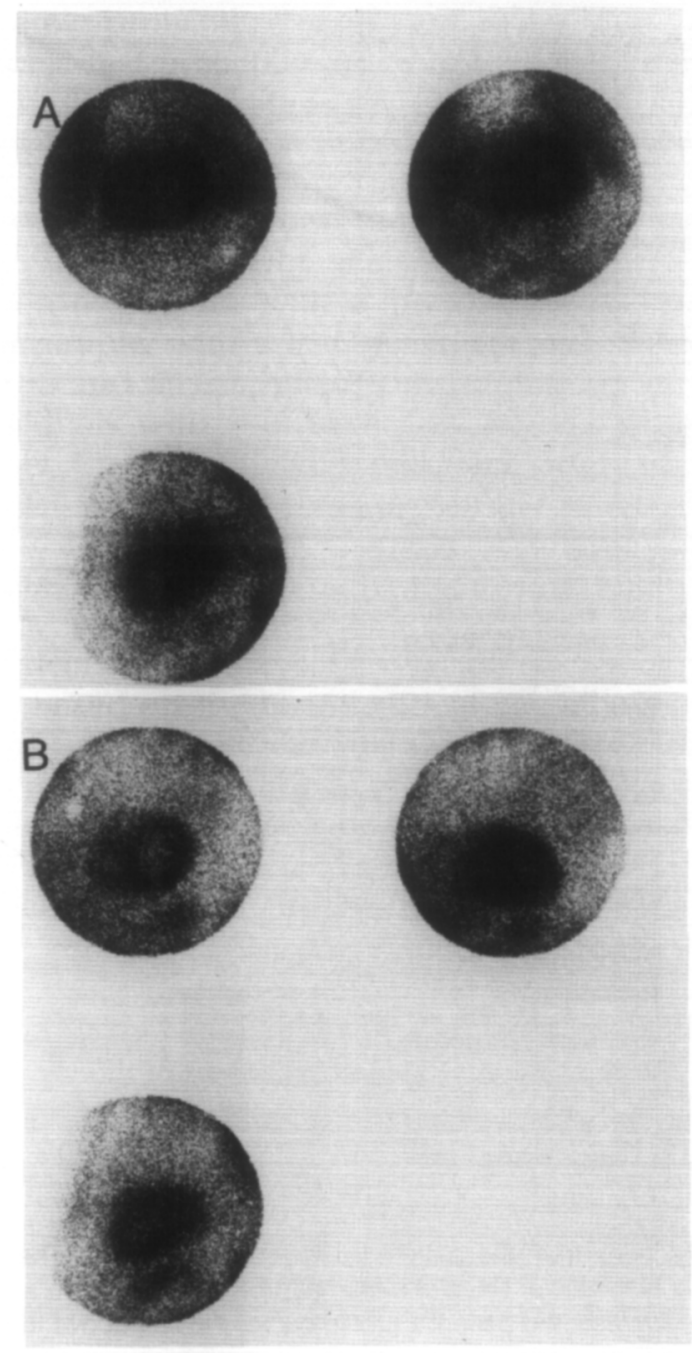

FIG 15.

A, immediate poststress images in a patient with a known prior inferior and posterior wall infarction. Note the greatly diminished activity seen in the inferior wall and posterior wall and manifested on all three views. (Top row, 45-degree left anterior oblique; hottom row, 70-degree left anterior oblique). B, corresponding 2-hour delayed images in the same patient reveal no fill-in or normalization of the defect. This is the classic pattern of a fixed or irreversible defect in an area of myocardial scar due to prior infarction. The early lung uptake on the poststress images that clears at the time of delayed imaging indicates some degree of congestive failure during exercise. Also note the. linear activity on the delayed left anterior oblique view due to "hang-up" of tracer in the arm veins.

with very low probabilities of coronary artery disease (Figs 16 and 17). ${ }^{57,58,79,81}$ Garcia and coworkers have described a unique extension of the quantification of ${ }^{201} \mathrm{Tl}$ tomographic images to create a polar map. ${ }^{80}$ In essence, the polar map depicts nested circumferential profiles from the apex to the base, which provides a means for presenting regional uptake data from an entire set of tomographic slices in one two-dimensional image.

The accuracy of quantitative analysis techniques remains under investigation. In one multicenter trial that compared circumferential profiles of 


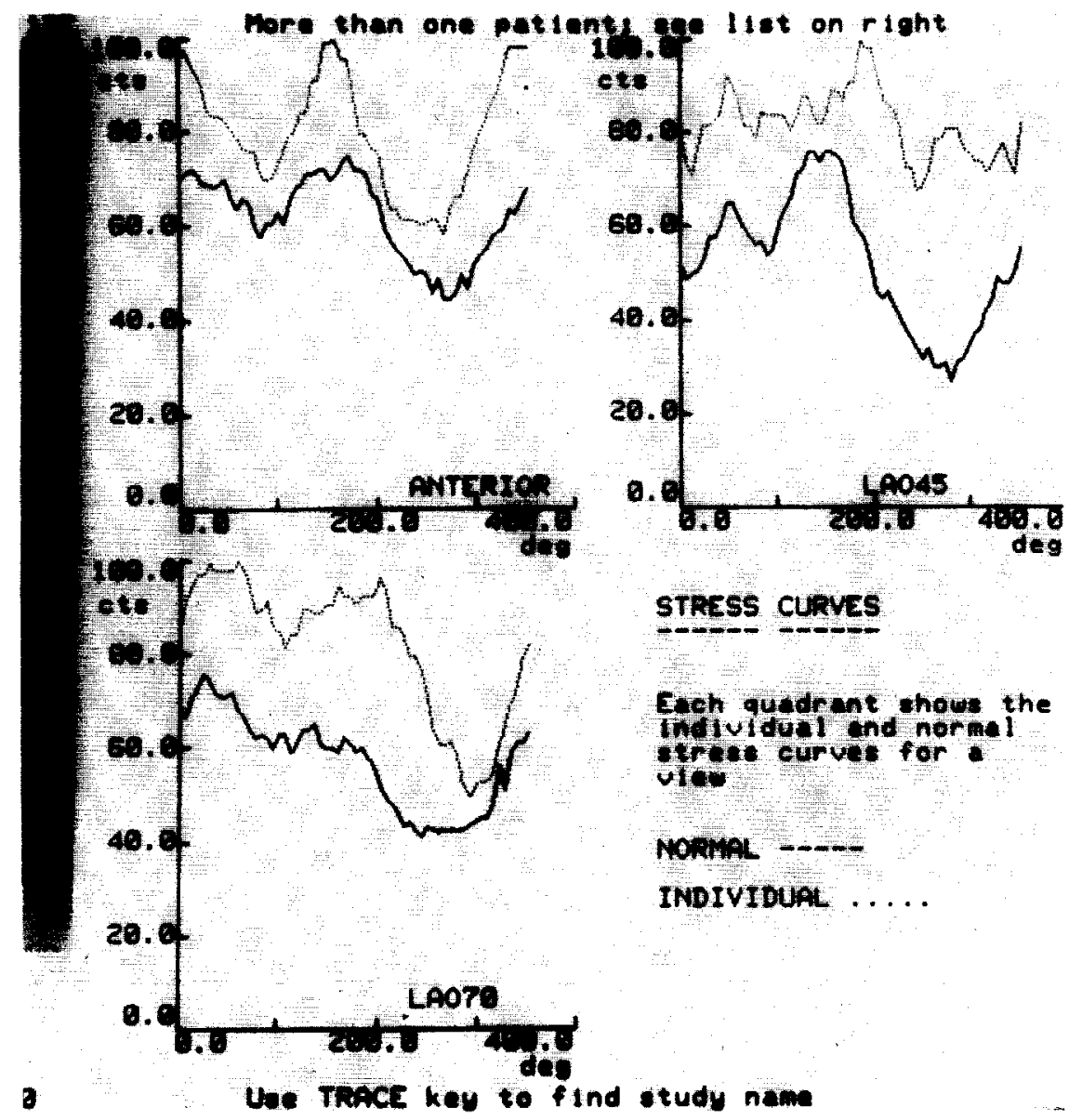

FIG 16.

Circumferential profiles in the 45- and 70-degree left anterior oblique projections from the patient illustrated in Figure 12. The upper curve in each panel represents data from the patient. The darker lower curve is the lower limits of a normal profile derived from a patient population with a $<1 \%$ probability of coronary artery disease. Note that in each view the patient's profile curve remains above the lower limits of the normal curve. Note also the significant variation in each view at different points around the perimeter.

stress and washout studies with statistically derived normal limits, the sensitivity for detection of coronary artery discase was $82 \%$ and the specificity was $83 \% .^{83}$ The quantitative techniques also permit a more detailed regional analysis of sensitivity and specificity in the major arterial distributions. The highest sensitivity in the study was obtained for the right coronary artery and left anterior descending artery systems, while the highest specificity was seen for the left circumflex artery. The low sensitivity for left circumflex artery disease detection is consistent with previous planar studies involving visual interpretation. ${ }^{83}$ The posterior location of the circumflex distribution permits evaluation in only one view by planar imaging.

In the current climate of increased awareness of medical costs and the need to assess the utility of diagnostic and therapeutic procedures, it is logical to try to identify those patients who might benefit most from stress ${ }^{201} \mathrm{Tl}$ imaging. From the standpoint of diagnosis only, patients with classic histories of angina pectoris have an $85 \%$ or greater probability of having anatomically significant coronary artery disease ${ }^{84}$ For these patients, a positive radionuclide stress test response would not alter the clinical decision to study the patient further, nor would a negative test result reduce the likelihood of disease to a low enough point to change the course of events diagnostically. Likewise, in patients with a very low pretest probability of disease, positive or negative noninvasive test results alone may not significantly alter patient management. It has been suggested in a number of analyses that the patient group with intermediate probabilities of disease $(30 \%$ to $70 \%)$ may benefit most from noninvasive testing ${ }^{85}$ In this range of probabilities, given the accuracy of ${ }^{201} \mathrm{Tl}$ scintigraphy, a positive or negative test result has a significant impact in determining the probability of disease. Patients with atypical chest pain, smoking history, obesity, hypertension or a family history of hypertension, but without classic angina often fall into this intermediate-probability category. Hamilton has calculated that (assuming a test sensitivity of $90 \%$ and a specificity of $80 \%$ ) for a patient with 
a pretest probability of disease in the $50 \%$ range the post-test probability rises to higher than $80 \%$ if the radionuclide study is positive and falls to lower than $15 \%$ if it is normal. ${ }^{85}$

The use of probability theory and, in particular, Bayes' theorem of conditional probahility further illustrates the difficulty of screening in a large population of patients with a low disease prevalence. For example, with a test sensitivity and specificity of $90 \%$ and $80 \%$ applied to a population of 1,000 individuals with a disease prevalence of approximately $5 \%$ (i.e., 50 with coronary artery disease and 950 without), 45 patients would have true positive test results, and 190 would have false-positive results. In this example, the positive predictive accuracy would only be $20 \%(45 / 235)$. If diagnosis and decision making for referral to angiography were based on the test results alone, a large number of patients without disease would be studied. This example illustrates the need to put each test result into the context of the overall clinical picture.

Pharmacological intervention has been pro- posed as an alternative to dynamic isotonic leg excrcise for coronary artery clisease detection by ${ }^{201} \mathrm{Tl}$ perfusion imaging. ${ }^{86,87}$ The preferred agent appears to be dipyridamole, although this drug is nol yet approved for routine clinical use by intravenous administration. Dipyridamole is a potent coronary vasodilator with minor systemic effects. Its use has been shown to cause a 3.5- to 4-fold increase in coronary blood flow together with a $20 \%$ to $40 \%$ increase in the heart rate and slight but significant decline in the blood pessure. The rationale for using dipyridamole in conjunction with ${ }^{201} \mathrm{Tl}$ scintigraphy is that the flow reserve of abnormal coronary arteries with fixed mechanical stenosis is more limited than is the flow reserve of normal vessels. Therefore, tracer delivery is selectively enhanced in the "watershed" of normal vessels, whereas regional areas of relative photopenia are seen in the distribution of diseased vessels that cannot respond to the drug.

The protocol for dipyridamole- ${ }^{201} \mathrm{Tl}$ scintigraphy involves the slow intravenous infusion of the drug over a 4-minute period at a total dose of $0.56 \mathrm{mg} /$

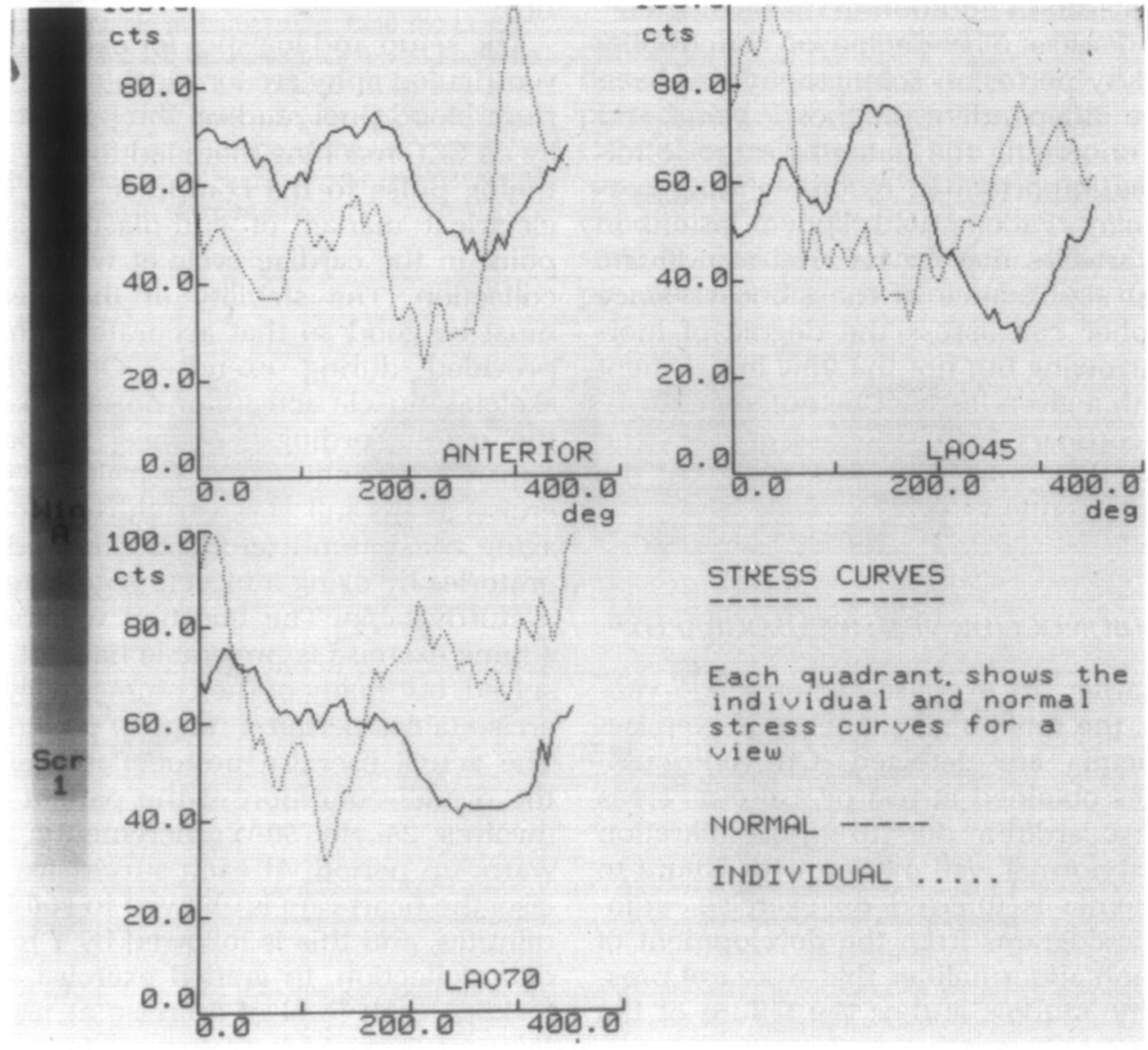

FIG 17.

Circumferential profiles from the patient illustrated in Figure 13 with significant coronary artery disease. Note that the patient's curve (iighter dotted curve) falls below the lower limits of a nomal curve on each view. All areas falling below the nurmal curve are regarded as demonstrating relative ischemia as compared with the area above the normal-limits curve. 
$\mathrm{kg}$. Tracer is injected 4 minutes after the infusion, and imaging is begun another 5 minutes thereafter. $^{86-88}$

The incidence of angina is lower with dipyridamole than with maximum dynamic exercise. Aminophylline is an antidote to dipyridamole and may be given in a dose of 50 to $150 \mathrm{mg}$ intravenously over 1 to 2 minutes for intervention-induced angina. ${ }^{88}$ The drug is otherwise generally well tolerated, although some patients experience headache, dizziness, or nausea.

The accuracy of the dipyridamole- ${ }^{201} \mathrm{Tl}$ technique is comparable to that of stress ${ }^{201} \mathrm{Tl}$ imaging. Reported sensitivities have ranged from $73 \%$ to $95 \%$ and specificities from $67 \%$ to $100 \% .{ }^{86-88}$

Some workers have attempted to augment the effect of dipyridamole by having the patient also perform handgrip isometric exercise at the time of tracer administration. The use of oral rather than intravenous dipyridamole has also been investigated. ${ }^{89}$ The onset of drug action is less predictable, and in the limited experience available for review, the oral approach appears less sensitive.

There are a number of reasons for performing a thallium stress scan in addition to diagnosing coronary artery disease. The degree of abnormality demonstrated by perfusion scintigraphy has been shown to have independent prognostic value, and information concerning the patient's exercise tolerance may be important in recommending specific management. Patients with known lesions in the coronary arteries may be referred to evaluate the functional significance of the stenoses since the angiographer can assess the degree of morphological narrowing but not the flow impairment associated with a given lesion. The outcome of angioplasty or coronary artery bypass grafting can also be assessed by imaging before and after the therapeutic intervention.

\section{EXERCISE RADIONUCLIDE VENTRICULOGRAPHY}

As noted earlier, in exercise radionuclide ventriculography, the functional sequelae of exerciseinduced ischemia are detected. On ventriculographic studies obtained at rest or exercise, areas of scarred myocardium due to prior infarction demonstrate abnormal wall motion with failure to contract. Ischemia is inferred on exercise radionuclide ventriculograms from the development of new wall motion abnormalities that were not present on baseline studies and/or the failure of the ejection fraction to increase.

The exercise radionuclide ventriculogram can be performed either as a first-pass (first-transit) study following a bolus injection of radiopharmaceutical or as an equilibrium gated blood pool procedure using ${ }^{99 m}$ Tc-labeled red blood cells. ${ }^{90,91}$ The advantages and disadvantages of the two approaches have been debated in the literature. The first-transit technique requires high-count rate instrumentation, and at least until recently, the count rate capability of standard gamma scintillation cameras has not been ideal for first-pass studies. Multicrystal detectors with a peak count rate capability of 200,000 counts/sec or more have been used successfully. Both approaches permit baseline studies and studies at increasing levels of exercise. Repeated injections of tracer are required for the first-transit studies. A major putative advantage of the first-transit approach is the rapidity of data collection to capture ventricular function at peak exercise. The equilibrium blood pool technique has the advantage of better counting statistics through imaging over a number of cardiac cycles but the disadvantage of being sensitive to cardiac arrhythmias and patient motion. The greater availability and versatility of the single-crystal gamma camera and the superior image quality of the gated equilibrium blood pool study have made this approach the more widely available in current practice.

The setup and logistics for exercise radionuclide ventriculography are formidable. For gated equilibrium blood pool studies, the patient is monitored by an ECG machine modified to provide an R-wave timing pulse to the computer. The R-wave is the electrical marker of end-diastole and a logical point in the cardiac cycle at which to begin data collection. The stability of the electrical signal IIlust be good so that accurate timing pulses are provided during exercise. Otherwise, spurious skeletal muscle activity or noise spikes can trigger the data-recording sequence. A special exercise apparatus is required to permit the patient to exercise to his full capacity during continuous imaging. Maximum exercise is achieved in most laboratories by using a bicycle ergometer attached to a sturdy table. The question whether upright or supine exercise is preferable has not been fully resolved, but many of the commercially available exercise tables permit a range of patient positioning. The actual exercise protocol is again tailored to the overall condition of the patient and typically involves 25- to 50- $W$ increments after a short warm-up period. At each successive level of exercise, the heart rate is allowed to stabilize for 1 to 2 minutes, and this is followed by 1 to 2 minutes of data collection. In graded exercise, it is desirable to keep each level of exercise at no more than 3 minutes to avoid leg fatigue prior to achievement of maximum exercise. Many laboratories record a postexercise study during the time when the heart is "unloaded" by peripheral vasodilation. The major technical limits to the procedure are faulty ECG 


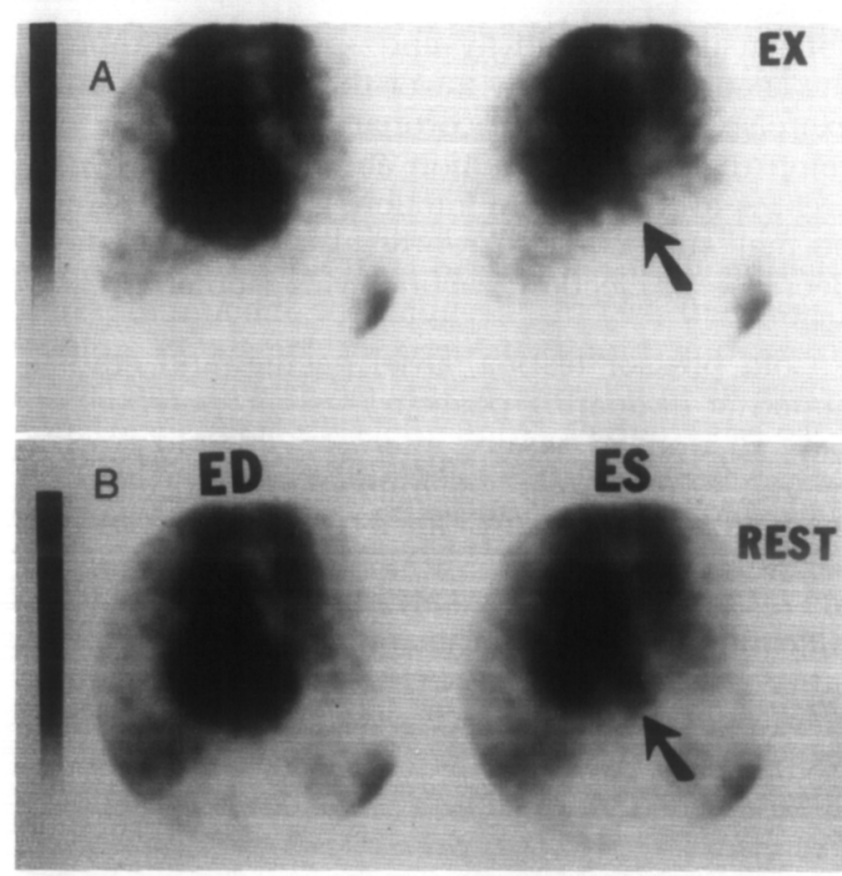

FIG 18.

A, end-diastolic and end-systolic images from a radionuclide ventriculogram obtained at rest in a normal subject. The arrow points to the apex of the left ventricle at end-systole. Note the uniform emptying of both the right and left ventricles between end-diastole and end-systole. B, corresponding end-diastolic (ED) and endsystolic (ES) views obtained at peak exercise. Note that the amount of tracer in the end-systolic image of the left ventricle is significantly less than on the resting study (arrow). This pattern illustrates the normal response to exercise.

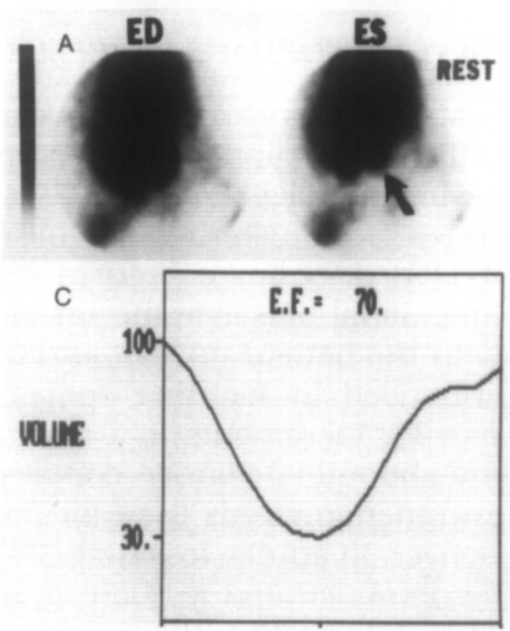

gating and excessive patient motion during exercise.

The analysis of exercise radionuclide ventriculograms includes an assessment of the baseline study and the studies obtained at each exercise level to determine the ejection fraction and assess regional wall motion. In normal subjects, wall motion should be uniform at rest, there should be no regional wall motion abnormalities apparent during exercise, and the ejection fraction should increase by $5 \%$ or more (Fig $18, A$ and $B$ ). In patients with significant coronary artery disease, wall motion abnormalities become manifest at high levels of exercise, and there is a failure of the ejection fraction to improve or even a decline in the ejection fraction (Fig 19,A-D) ${ }^{90}$ Various investigators have suggested measurement of other quantitative parameters such as the rate of ventricular emptying and filling $(\mathrm{d} V / \mathrm{d} t)$. Analytic interest has also focused on parametric analysis (phase analysis) to detect subtle areas of ventricular asynnergy not seen on the closed-loop cinematic display. These alternative methods have nol yet become useful in routine clinical practice.

The accuracy of exercise radionuclide ventriculography has been studied in a number of institutions. Sensitivities of $85 \%$ to $95 \%$ have been reported and specificities from $54 \%$ to $100 \%{ }^{90-95}$ Careful examination of the literature in this area reveals that varying patient populations, study conditions, and diagnostic criteria make comparisons of clinical research data difficult. For example, Borer and coworkers, who first described the

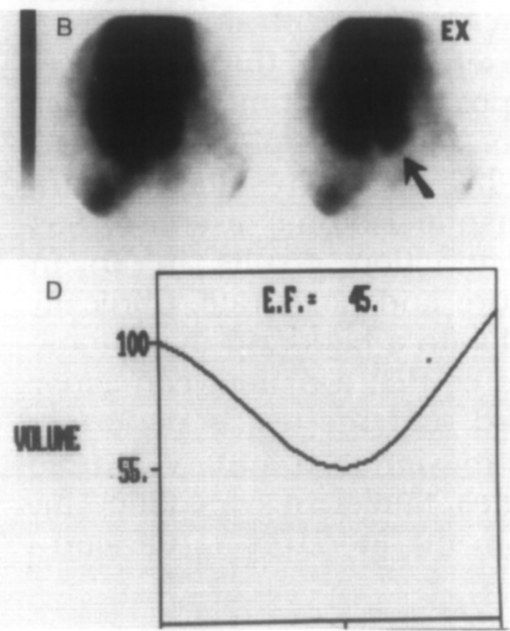

\section{FIG 19.}

A, end-diastolic (ED) and end-systolic (ES) images obtained at rest in a patient with significant coronary artery disease. No abnormality of ventricular function is apparent. The left ventricle is emptied uniformly and well between end-diastole and end-systole (arrow). B, corresponding end-diastolic and end-systolic images obtained during peak exercise reveal a failure of the ventricular emptying to improve. The amount of activity remaining in the left ventricle at end-systole (arrow) is far greater than that at rest and indicates exerciseinduced ventricular dysfunction due to exercise-induced ischemia. The right ventricle continues to contract normally. $\mathbf{C}$ and $\mathbf{D}$, timeactivity or time-volume curves obtained at rest (C) and exercise (D) reveal a significant dectine in the ejection fraction (E.F.) from $70 \%$ to $45 \%$. In normal subjects, the ejection fraction should increase in response to exercise. 
technique, used supine bicycle exercise and discontinued treatment with antianginal drugs for the procedure. ${ }^{90,93}$ Jones and coworkers reported on patients exercised upright who continued to take their cardiac drugs..$^{91}$ In some series, patients with abnormal baseline studies have been included, a practice that tends to increase the apparent sensitivity of the procedure. Another issue is whether a given series included patients unable to achieve maximum exercise. Monitoring and documentation of the total length of exercise, external work load (ergometer setting), pressure-rate product (blood pressure and heart rate), ECG response, and patients' symptomatic responses have been highly variable in reported series. The significance of exercise level is well illustrated in Brady and coworkers' series. ${ }^{94}$ The sensitivity for detecting coronary artery disease was $95 \%$ in patients who exercised to the point of chest pain, the development of 1.0mm ST segment depression or more, or a pressure-rate product (systolic blood pressure times heart rate) of 25,000 or greater. The sensitivity in patients failing to achieve any of these criteria was $62 \%(P<.02)$. In critically evaluating the literature, not only for exercise radionuclide ventriculography but for all forms of stress testing, these factors must be kept in mind. In clinical practice, decisions must be made for every patient, including those who cannot achieve adequate exercise or who cannot be weaned from their cardiac medications.

\section{ALTERNATIVES TO LEG EXERCISE}

Table 3 provides a summary of alternatives to treadmill or bicycle exercise. All of these have been explored for the purpose of detecting coronary artery disease. None of the other physiological or physical techniques has shown results comparable to those of dynamic isotonic exercise. Sustained isometric contraction results in an increased blood pressure and heart rate. Clinically, the test is performed with a handgrip dynamometer. The maximum force that a patient can generate is first determined, and the test is performed at $25 \%$ to $33 \%$ of the maximum voluntary contraction for 3 to 5 minutes. Sufficient experience has not been presented in the literature to develop a

\section{TABLE 3.}

Alternative to Dynamic (Leg) Exercise

Isometric (handgrip) exercise
Cold pressor testing
Atrial pacing
Postextrasystolic potentiation
Pharmacological stress (catecholamines)

consensus of sensitivity and specificity, although the accuracy is clearly lower than that of dynamic exercise. Patients with coronary artery disease develop regional wall motion abnormalities and decreases in the ejection fraction. ${ }^{96,97}$ However, some normal subjects apparently also demonstrate a decrease in ejection fraction with isometric exercise.

Cold pressor testing, atrial pacing, and studies aimed at evaluating postextrasystolic potentiation have all aroused passing interest. ${ }^{98-102}$ Cold pressor testing is performed by immersing one hand in ice-cold water $\left(0\right.$ to $\left.2^{\circ} \mathrm{C}\right)$ for 2 to 3 minutes. In two series reported by Manyari et al. and by Jordan et al., respectively, the sensitivity of this test was significantly less than for dynamic exercise ${ }^{98,99}$ In the latter series, only 24 of 41 patients $(57 \%)$ demonstrated an abnormal response to cold pressor testing as compared with 34 of the $42(81 \%)$ demonstrating wall motion abnormalities during dynamic exercise. $^{99}$

\section{GASTROINTESTINAL STUDIES}

Diagnostic studies using radionuclide techniques have been developed to detect gastroesophageal reflux and to measure esophageal and gastric function. The unique ability to quantitatively measure the passage of a radioactive tracer through the luminal structures of the gut has afforded the ability to assess functional change in response to drugs for both diagnostic and therapeutic purposes.

\section{GASTROESOPHAGEAL REFLUX}

Malmud and Fischer have demonstrated the utility of radionuclide techniques for detecting gastroesophageal reflux. ${ }^{103-105}$ The procedure involves administering $0.5 \mathrm{mCi}$ of ${ }^{99 \mathrm{~m}} \mathrm{Tc}$-pentetate (DTPA) or sulfur colloid in $300 \mathrm{ml}$ of isotonic saline. Serial 30-sec/frame images are obtained, first with the patient upright and then supine. Reflux is enhanced by exerting epigastric pressure in 20 $\mathrm{mm} \mathrm{Hg}$ increments to a maximum of $100 \mathrm{~mm} \mathrm{Hg}$. An abdominal binder containing a pressure cuff and manometer is used for this interventional maneuver. In adults, less than $5 \%$ of the tracer should be demonstrated in the esophagus. In addition to diagnosing reflux, the technique can be used to assess the effect of pharmacological agents such as bethanechol, a cholinergic agonist that increases the lower esophageal sphincter tone and reduces reflux. Metoclopramide has some cholinergic agonist effects and may also reduce reflux. The efficacy of agents such as alginic acid, which prevent reflux by forming a barrier layer over the stomach 
contents, is also readily evaluated by the radionuclide technique. ${ }^{106}$

\section{GASTRIC EMPTYING}

The physiology of gastric motility and the rates of gastric emptying have been extensively studied with radionuclide techniques. ${ }^{107}$ In fact, the investigation of agents to simulate liquid and solid meals has almost become a subculture in radiopharmaceutical development. ${ }^{108}$ Perhaps the earliest and most exotic radiopharmaceutical to be evaluated for this purpose was radiolabeled chicken liver. ${ }^{109}$ The procedure involved injecting ${ }^{99 m} \mathrm{Tc}$-sulfur colloid into a live chicken followed by sacrifice of the chicken after the tracer had been taken up in the liver. The radiolabeled liver was then cooked and fed to the patient. This in vivo labeling method guaranteed that the radionuclide remained attached to the parent material, but as might be anticipated, patient and laboratory acceptance was not uniformly good, thus prompting the development of alternative radiotracers for the analysis of gastric emptying. The most widely accepted agents for this purpose in current practice are ${ }^{99 \mathrm{~m}} \mathrm{Tc}$-sulfur colloid-labeled eggs for the investigation of solid gastric emptying and ${ }^{99 m} \mathrm{\Gamma c}-$ or ${ }^{111}$ In-pentetate (DTPA) for liquid emptying studies.

As with studies of gastroesophageal reflux, a central interest in gastric emptying studies is the

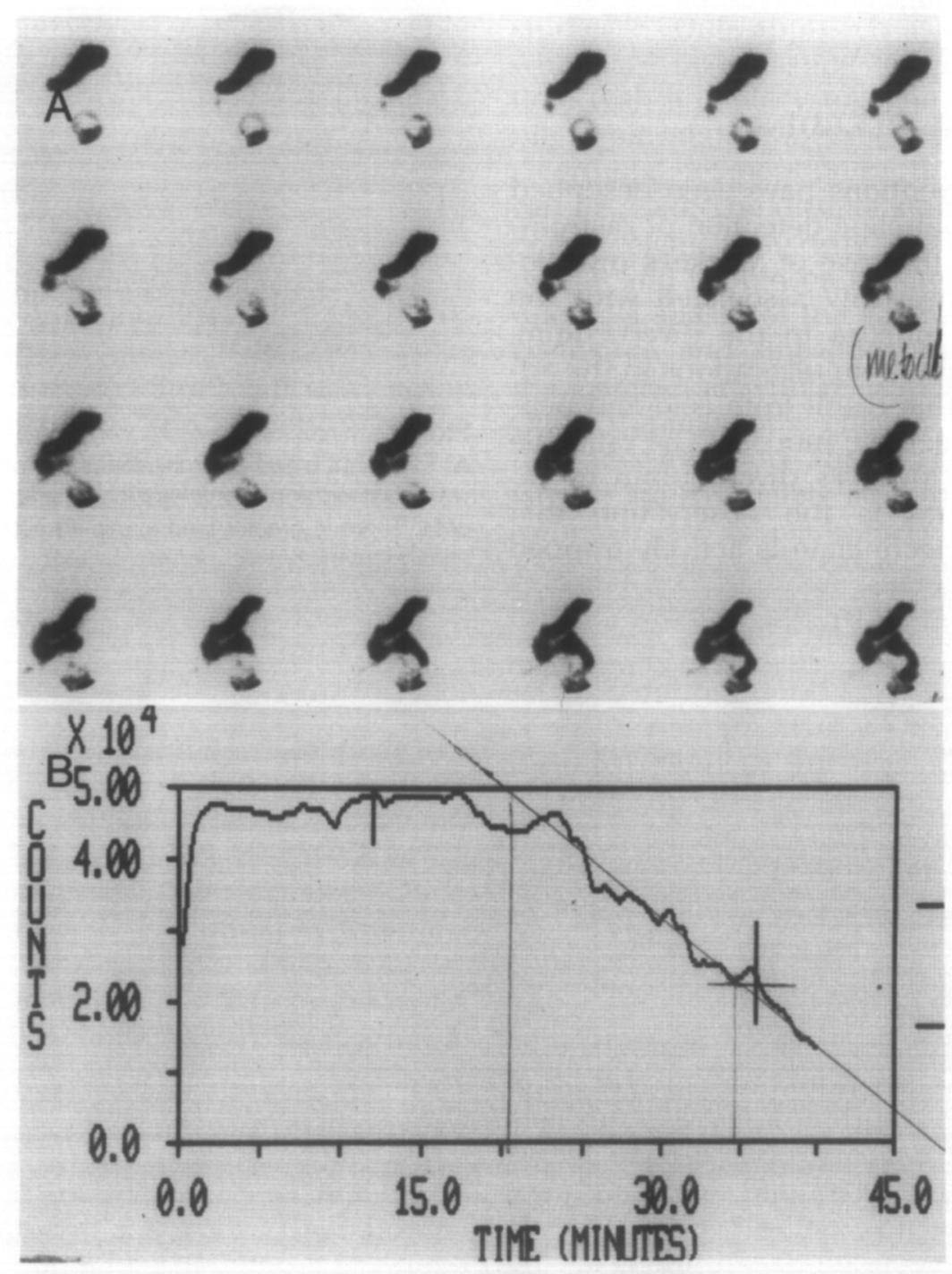

FIG 20.

A, sequential 1 -minute images from a gastric emptying study obtained with a liquid meal. Very little activity has left the stomach and entered the small bowel during the first 12 images (top two rows). The patient received metoclopramide at approximately 12 minutes. After a few minutes' delay, a significant acceleration is demonstrated with tracer entering the small bowel. This pattern illustrates a positive response to metoclopramide and confirms the likelihood of therapeutic efficacy of the drug in this patient. B, corresponding time-activity histogram reveals essentially no emptying during the premetoclopramide phase of the study. The curve is flat. After a few minutes' delay, there is a marked downsloping of the curve that corresponds to the observed emptying of tracer into the small bowel. 
response of the physiological process to various pharmacological interventions. For example, metoclopramide is used to promote gastric emptying in diabetics with gastroparesis. The success of this therapy depends, however, on some preservalion of cholinergic activity. When using nuclear techniques, the rate of gastric emptying can be measured before and after the administration of metoclopramide intravenously as a predictor of response to chronic oral therapy (Fig 20,A and B) ${ }^{110}$ The side effects of metoclopramide are significant enough that a rational demonstration of the therapeutic effect is valuable in deciding whether to continue or discontinue therapy. This is just one example of literally dozens illustrating the use of pharmacological interventions in studying gastric physiology.

\section{GASTROINTESTINAL BLEEDING}

A number of interventions have been described to enhance the radionuclide detection of gastrointestinal bleeding. In the case of Meckel's diverticulum, bleeding is invariably associated with the presence of gastric mucosa in the diverticulum. The bleeding is usually from ulcerations in the adjacent ileal mucosa that result from acid and pepsin secretion by the ectopic mucosa. The rationale for the detection of Meckel's diverticulum by radionuclide techniques is the observation that ${ }^{99 \mathrm{~m}} \mathrm{Tc}$ as sodium pertechnetate is actively trapped and secreted by the gastric mucosa. ${ }^{111}$ The imaging technique involves administering $10.0 \mathrm{mCi}{ }^{99 \mathrm{~m}} \mathrm{Tc}-$ pertechnetate intravenously followed by imaging of the patient's abdomen at 5 -minute intervals for 1 hour. ${ }^{112}$ Pentagastrin, which increases gastric blood flow and stimulates the secretion of acid, has been used to enhance the localization of ${ }^{99 \mathrm{~m}}$ Tc-pertechnetate in Meckel's diverticulum. ${ }^{113-115}$ Although significant clinical data are lacking, cases have been reported in which an initial failure to detect a Meckel's diverticulum was rectified by administering pentagastrin and repeating the study. ${ }^{115}$

Another interesting enhancement method used adjunctively in the detection of gastrointestinal bleeding is the administration of glucagon to paralyze the gut. ${ }^{116}{ }^{99 \mathrm{~m}} \mathrm{Tc}$-labeled sulfur colloid and red blood cells have demonstrated significant clinical efficacy in detecting active gastrointestinal bleeding; however, accurate localization of the bleeding site may be problematic due to peristaltic activity and movement of radiotracer away from its original site of deposition. For bleeding sites in the small intestine, the intensity of peristaltic activity and rapidity of tracer disbursal may actually result in a false-negative study or a study demonstrating only diffuse activity. By paralyzing the gut with

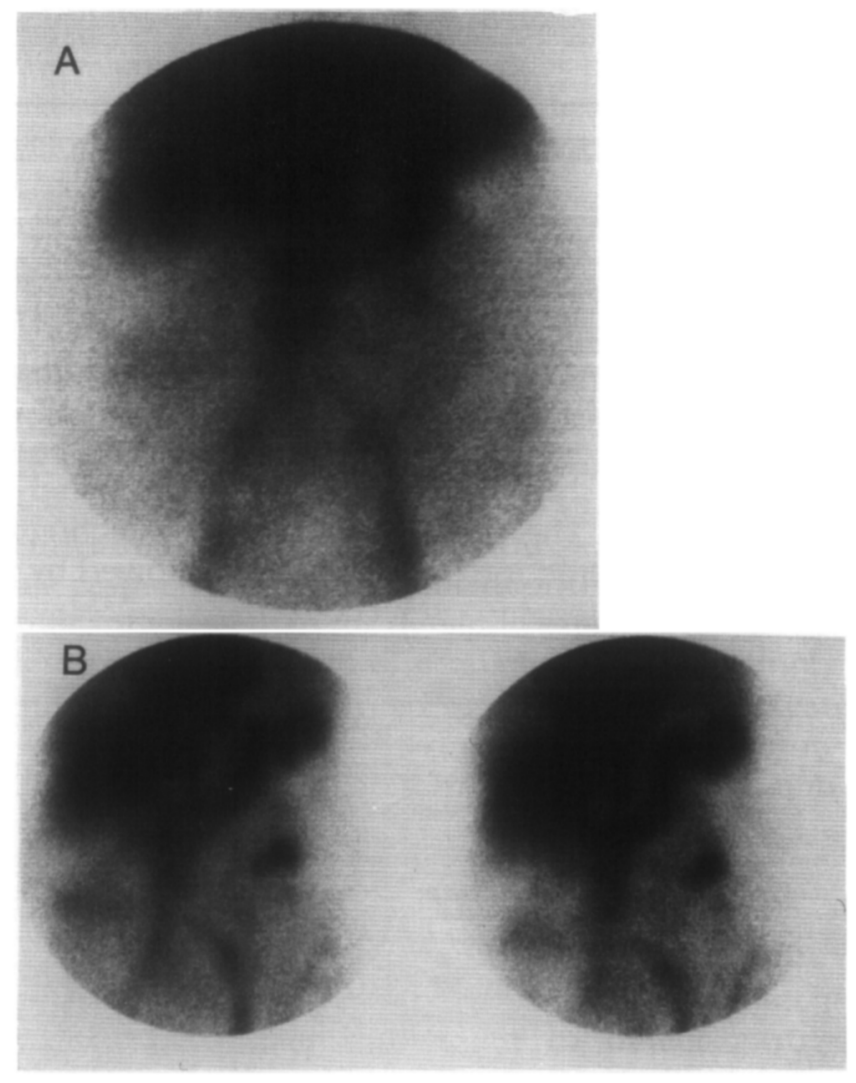

FIG 21.

A, ${ }^{99 m}$ Tc-red blood cell gastrointestinal bleeding study. The image was obtained approximately 2 hours after administration of the red cells. There is greater than expected activity in the left part of the midabdomen, but no definite localized bleeding site is demonstrated. B, following the administration of glucagon and continued imaging at 10-minute intervals, an unequivocal focal area of tracer localization is demonstrated in the left part of the midabdomen laterally

glucagon, the radiotracer is allowed to pool at the bleeding site, thus enhancing its localization and the lesion's detectability (Fig 21,A and B). A dose of 0.5 to $1.0 \mathrm{mg}$ of glucagon is administered intravenously followed by sequential imaging using a standard 5-minute-per-view format. The effect of the glucagon diminishes after 30 minutes, and if suspicion of active bleeding remains high, an additional dose may be administered.

\section{HEPATOBILIARY IMAGING}

Hepatobiliary interventions have become important adjuncts to imaging in both the pediatric and adult age groups. In pediatric practice, hepatobiliary imaging with ${ }^{131} \mathrm{I}$-rose bengal or, more recently, ${ }^{9.9 m^{\prime}}$ Tc-iminodiacetic acid (IDA) derivatives (e.g., disofenin, merbrofenin, lidofenin) is used to distinguish neonataf hepatitis from biliary atresia. However, rose bengal and the IDA derivatives compete with bilirubin for the hepatic organic anion excretion pathway, and false-positive studies may 
result in some cases of abnormally high bilirubin levels (Fig 22,A and B). Phenobarbital stimulates overall hepatic transport and excretory functions and thereby represents a useful intervention to promote the hepatocyte excretion of these radiotracers. ${ }^{117,118}$ The suggested dose of phenobarbital is $5 \mathrm{mg} / \mathrm{kg}$ of body weight per day in divided doses for at least 5 days before the ${ }^{99 m}$ Tc-IDA study.

Majd and colleagues reported prephenobarbital and postphenobarbital studies in 16 patients. The initial study demonstrated no hepatobiliary excretion of the ${ }^{99 m}$ TC-IDA derivative in any patient. ${ }^{119,120}$ Six of these patients demonstrated excretion following phenobarbital administration. Nine of the ten remaining subjects were shown to have biliary atresia at surgery. Anecdotal experience in smaller series has supported these results.

The principal clinical use of the ${ }^{99 m}$ Tc-IDA derivatives is in the differential diagnosis of abdominal pain, specifically in patients with suspected acute cholecystitis. ${ }^{121,122}$ Unequivocal visualization of the gallbladder essentially rules out acute cholecystitis except in a small percentage of patients with acalculous cholecystitis in whom the gallbladder is visualized. The predictive accuracy of a negative study is high enough to alter clinical decision making from "surgery" to "no surgery." ever, the predictive accuracy of positive results, i.e. failure to visualize the gallbladder, is much lower in clinical situations in which the flow of bile into the gallbladder is diminished. ${ }^{124}$ In patients who have been fasting for prolonged periods of time or who are undergoing hyperalimentation, the gallbladder is not stimulated to contract; there is a resultant increase in bile viscosity and a stasis of bile flow. In this situation, gallbladder entry of the hepatobiliary radiotracers is restricted. Numerous investigators have shown a high incidence of falsepositive hepatobiliary studies in chronically ill, debilitated, and hospitalized patients, probably for this reason.

Since the phenomenon of impaired biliary kinetics is somewhat predictable from the patient's history, strategies have been developed to convert potentially false-positive studies to true negative studies. ${ }^{125}$ One approach is to pretreat patients who have been fasting for prolonged periods of time with a cholecystogogue. Either cholecystokinin or sincalide (Kinevac), a drug consisting of the C-terminal octapeptide portion of cholecystokinin, can be used. The dose of sincalide is $0.02 \mu \mathrm{g} / \mathrm{kg}$ of body weight. The drug is administered intravenously and should be injected slowly to avoid inducing a spasm of the gallbladder neck. ${ }^{125}$ Cholecystokinin and sincalide produce contraction of the gallbladder with relaxation of the sphincter of Oddi. The maximum effect is reached 5 to $15 \mathrm{~min}$ utes after injection. The ${ }^{99 \mathrm{~m}} \mathrm{Tc}$-IDA agent is administered approximately 30 minutes after the chole-
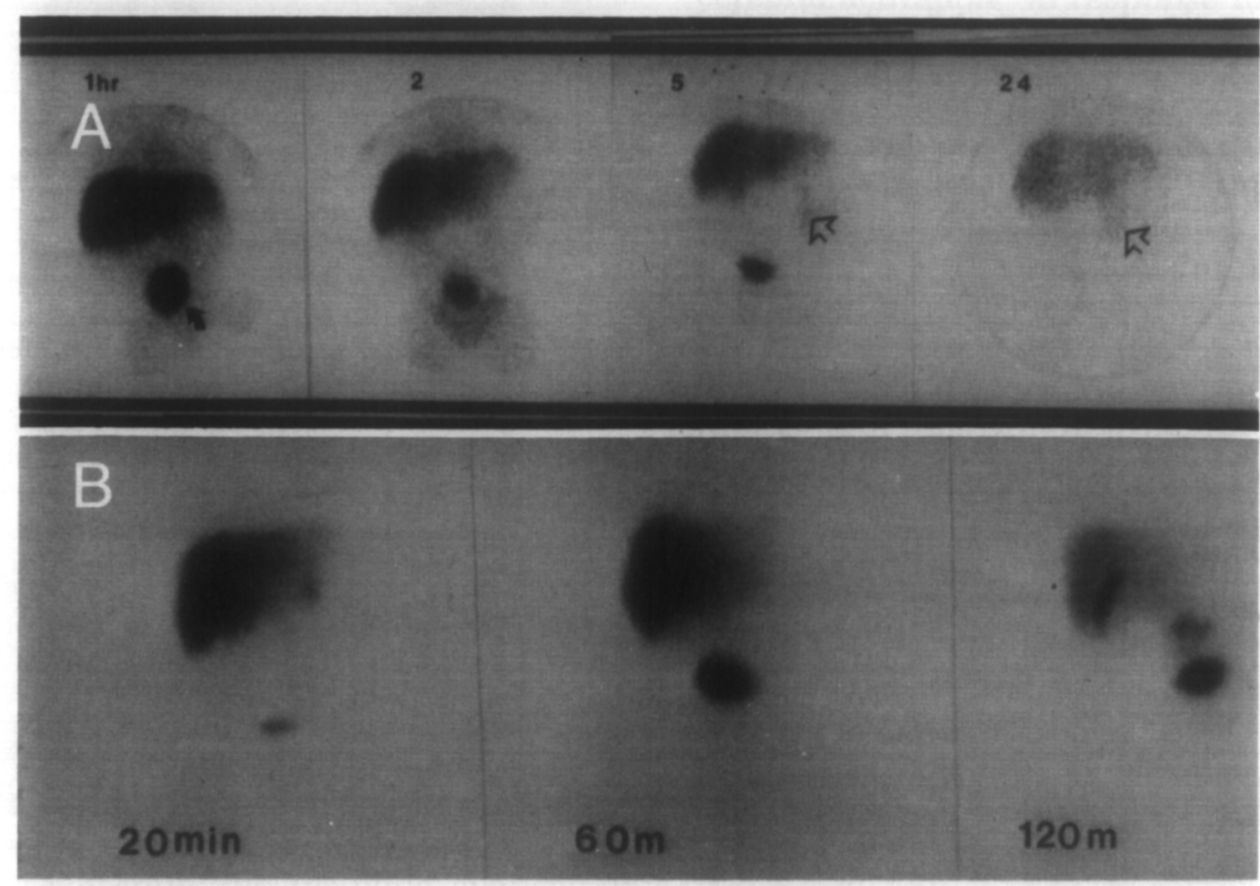

FIG 22.

A, ${ }^{99 m} \mathrm{Tc}$-IDA imaging in a patient with suspected congenital biliary atresia. Imaging without pretreatment with phenobarbitol ta:ls to reveal excretion of activity into the gut. The bladder (closed arrow) and the kidneys (open arrows) are visualized and should not be mistaken for tracer activity in the gut. B, following pretreatment with phenobarbital, the paticnt now demonstratcs uptake in the gallibladder and excretion into the gut. 
cystokinin or sincalide administration so that the maximum excretion of the radiotracer into the biliary system occurs during the refilling phase of the gallbladder. Sincalide is usually well tolerated. Patients may complain of abdominal discomfort and the urge to defecate, and occasionally nausea, dizziness, or flushing may occur.

It has recently been noted that pretreatment with sincalide may delay the time of visualization of the tracer in the small bowel, possibly causing a misdiagnosis of common bile duct obstruction. The pathophysiology of this observation has not been established but may relate to diversion of bile to the gallbladder during its filling phase.

An alternative to the pretreatment approach is to administer the ${ }^{99 \mathrm{~m}} \mathrm{Tc}$-IDA agent and image sequentially in the usual manner over a period of 1 to 2 hours. If the gallbladder is visualized, no interventional maneuver is required. If the gallbladder fails to be visualized, it is possible to administer the cholecystogogue, and after an appropriate 30-minute interval, a second dose of the ${ }^{99 m_{T}}$ Tc-IDA agent is injected (Fig 23,A and B). ${ }^{125}$ The choice of which approach to use is obviously at the discretion of each institution, although pretreatment intervention results in shorter study times.

An interesting alternative intervention proposed for the enhancement of gallbladder visualization is the administration of intravenous morphine. The rationale is that morphine causes constriction of the sphincter of Oddi with a resultant increase in the pressure in the common bile duct and increased flow of bile and radiotracer into the gallbladder. The technique is applicable if tracer is clearly demonstrated in the common duct. The dose of morphine sulfate is $0.04 \mathrm{mg} / \mathrm{kg}$ of body weight and is administered intravenously over 3 minutes. In the original series by Choy et al., the specificity of hepatobiliary imaging improved from $83 \%$ to $100 \%$ with the morphine intervention. ${ }^{126}$ Interestingly, morphine-induced contraction of the sphincter of Oddi may give the false impression of common bile duct obstruction in patients receiving this drug for the control of pain. As for all other procedures where incidental drug therapy may alter the test results, patients undergoing ${ }^{99 m}$ Tc-IDA imaging should be questioned for drug histories, especially for the use of morphine or morphinelike products (Fig 24,A and B).

In the early experience with ${ }^{99 m}$ Tc-IDA hepatobiliary imaging, there was concern that acalculous cholecystitis could cause false-negative results. ${ }^{122}$ Some authors suggested that as many as $4 \%$ of patients presenting with cholecystitis might fall into this category. The phenomenon is apparently a real one but has not proved to be common or clinically important. Nonetheless, if the gallbladder is visualized and there is still a high degree of clinical

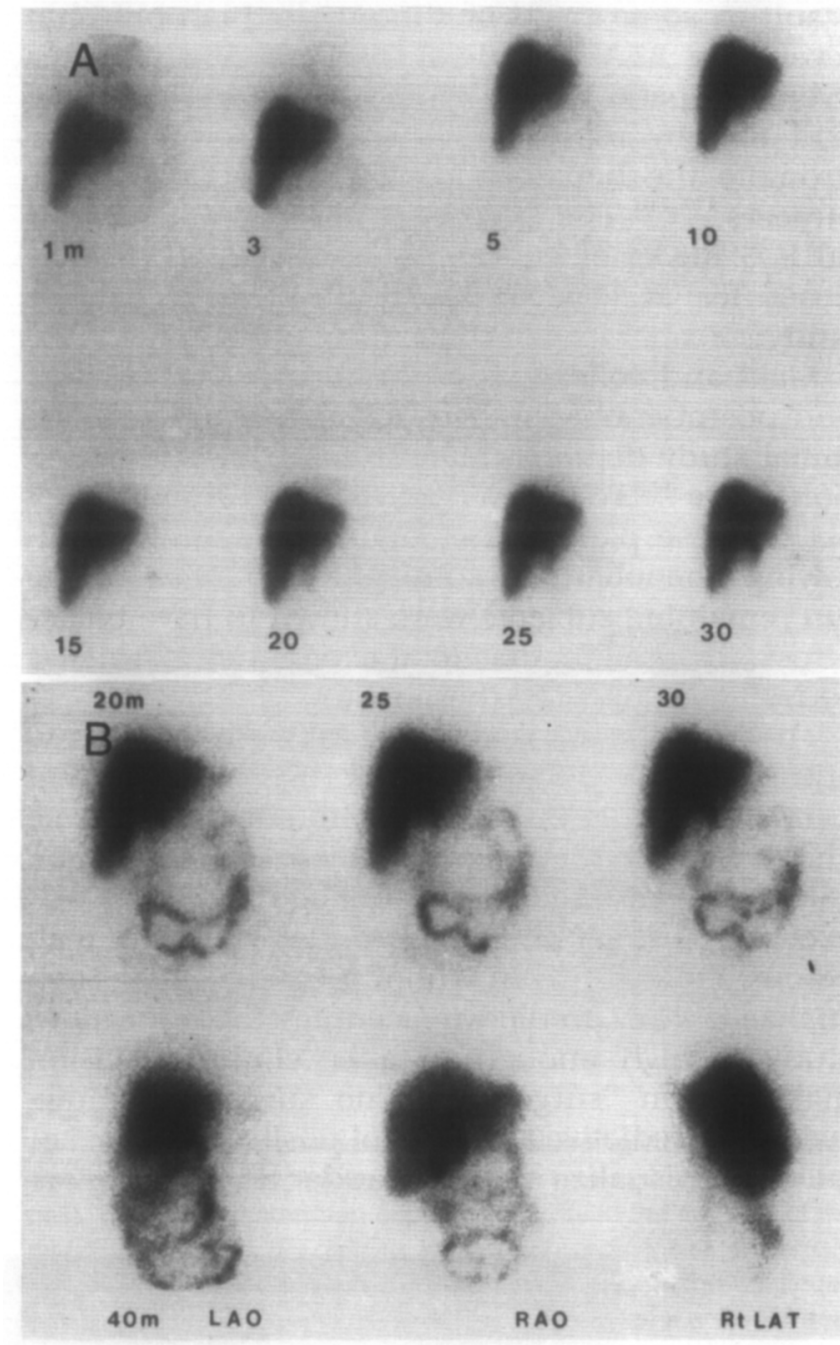

FIG 23.

A, sequential images from a ${ }^{99 m} \mathrm{TC}-$ IDA study in a patient with suspected acute cholecystitis. No uptake is demonstrated in the gallbladder. Imaging was continued for another 90 minutes without visualization of the gallbladder. B, reinjection of ${ }^{99 m} \mathrm{TC}-I D A$ was performed 20 minutes after treatment with sincalide. Note the absence of gallbladder activity on the 20-, 25-, and 30-minute images. The gallbladder is unequivocally demonstrated on the 40minute right anterior oblique view. The use of sincalide in this case converted a false-positive study to a true negative study.

suspicion that the patient has acalculous cholecystitis, a cholecystogogue may be administered Since the normal gallbladder responds immediately and vigorously to cholecystokinin or sincalide, such a response rules out acalculous cholecystitis. ${ }^{122}$ The inflamed, edematous gallbladder wall in cases of cholecystitis does not respond or responds poorly to this intervention.

A limited number of centers have reported another potential use for cholecystogogues in the evaluation of patients presenting with persistent postprandial right upper quadrant pain and biliary colic but with normal study findings of the gallbladder by oral cholecystography, ultrasound, or 


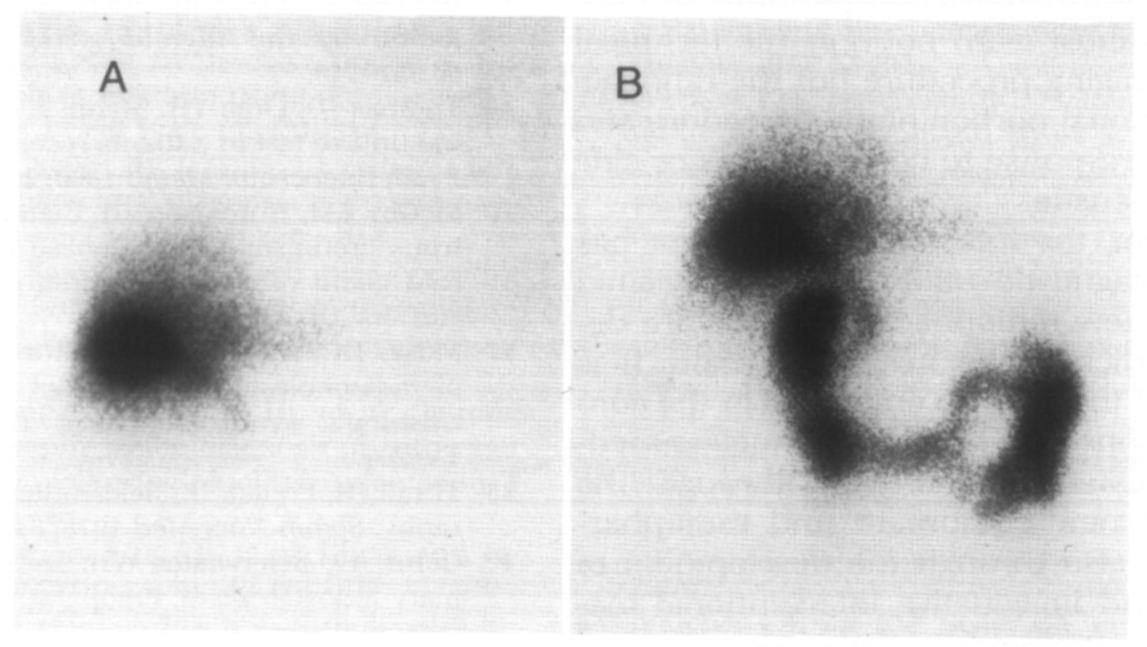

FIG 24.

A, anterior view of a ${ }^{99 m}$ TC-IDA study obtained 120 minutes after tracer administration. There is intense tracer accumulation in the gallbladder with visualization of the common duct but no activity in the small bowel. The patient was receiving morphine for the control of pain. Failure to visualize the small bowel at $\nmid 20$ minutes is distinctly abnormal and raises the possibility of a common bile duct obstruction. B, a repeat image obtained 20 minutes after the administration of sincalide reveals significant excretion of tracer into the duodenum and proximal jejunum, thereby ruling out a common duct obstruction but illustrating the potential for false-positive interpretation unless a detailed drug history is elicited

upper gastrointestinal series. ${ }^{125,127}$ This symptomatology is felt to be due to noncalculous partial cystic duct obstruction. ${ }^{128}$ The rationale for the use of cholecystokinin or sincalide is that patients with abnormal cystic ducts as a cause of their symptoms demonstrate a diminished gallbladder ejection fraction. The gallbladder ejection fraction is calculated by determining the net count rate in the gallbladder before cholecystogogue administration and at specified time intervals thereafter. In Fink-Bennett and colleagues' series, no patient with a pathologically confirmed abnormality of the cystic duct had an ejection fraction exceeding $22 \% .{ }^{127}$ The percentage of tracer expelled from the gallbladder should exceed $35 \%$ at 20 minutes in normal subjects.

\section{CENTRAL NERVOUS SYSTEM INTERVENTIONS}

Interventional studies of the central nervous system have not yet become extensively used clinically but have figured significantly in research aimed at understanding the relationship between cerebral anatomy, function, and metabolism. The brain has evolved so that specific anatomic regions in the cortex are responsible for effecting specific functions. As these regions are activated by physical or cognitive stimuli, the resulting changes in blood flow and metabolism can be directly measured by radionuclide techniques. The most extensively used radiopharmaceutical for such metabolic studics is ${ }^{18} \mathrm{~F}$-fluorodeoxyglucose. Kinetic models have been developed that permit the ac- tual measurement of the glycolytic rate on a pergram-of-tissue basis. ${ }^{129-131}$ The current assumption is that the rate of glucose utilization directly reflects functional activity since it is the essential metabolic substrate in the brain.

Elegant studies have been performed to map "cerebral activation" in response to physical stimuli or cognitive paradigms. ${ }^{132-135}$ For example, a baseline study of regional cerebral blood flow or glucose metabolism may be first obtained in a quiet darkened room with the patient's eyes closed. Cortical activation in response to visual or auditory stimuli or to complex scenes then permits a direct demonstration of which regions of the brain are processing these stimuli.

More recently, studies of the central nervous system have been extended to include a variety of receptor-binding radiopharmaceuticals. ${ }^{136,137}$ These agents are being used to probe the status of receptor activity and binding capacity. Pretreatment or post-treatment with "cold" receptor-binding drugs can significantly alter the radiotracer uptake patterns and may provide inferences on binding capacity and the relative affinity of receptors in different areas.

\section{CONCLUSION}

Interventional studies have several important broad implications for the practice of nuclear medicine. First, interventional studies are typically more complex than are noninterventional studies. They involve administering polent drugs and performing physiological maneuvers that may elicit 
significant symptoms. Physicians performing these types of studies must learn not only the technical details of the imaging procedure but the rationale for the interventional portion of the procedure and the appropriate response to complications or side effects that may ensue.

Most important, the interventional concept has extended the diagnostic utility of the associated procedures. As new radiopharmaceuticals are developed for studies of the heart and brain, it is highly likely that they will be employed in the context of interventions. The horizons of nuclear medicine will be extended not only through the development of new equipment and radiopharmaceuticals but also through the development of new, creative, and imaginative interventional maneuvers.

\section{REFERENCES}

1. Thrall JH, Swanson DP: Interventional aspects of nuclear medicine, in Freeman LM, Weissman HS (eds): Nuclear Medicine Annual 1983. New York, Raven Press, 1983, pp 1-50.

2. Thrall JH, Swanson DP: Diagnostic Interventions in $\mathrm{Nu}$ clear Medicine. Chicago, Year Book Medical Publishers, Inc, 1985.

3. Spencer RP: Interventional Nuclear Medicine. Orlando, Fla., Grune \& Stratton, 1984.

4. Werner SC, Spooner M: A new and simple test for hyperthyroidism employing 1-triiodothyronine and the 24-hour $\mathrm{I}^{131}$ uptake method. Bull NY Acad Med 1955; 31:137-145.

5. Sisson JC: Interventions in patients with thyroid disease, in Thrall JH, Swanson DP (eds): Diagnostic Interventions in Nuclear Medicine. Chicago, Year Book Medical Publishers, Inc, 1985, pp 118-123.

6. Hershman JM: Clinical application of thyrotropin-releasing hormone. $N$ Engl $J$ Med 1974; 290:886-890.

7. Clifton-Bligh P, Silverstein GE, Burke G: Unresponsiveness to thyrotropin-rcleasing hormone (TRH) in treated Graves' hyperthyroidism and in euthyroid Graves' disease. $J$ Clin Endocrinol Metab 1974; 38:531538 .

8. Hamburger JI: Solitary autonomously functioning thyroid lesions. Am J Med 1975; 58:740-748.

9. Hurley JR, Becker DV: Thyroid suppression and stimulation testing: The place of scanning in the evaluation of nodular thyroid disease. Semin Nucl Med 1981; 11:149-160.

10. Benua RS, Sonenberg $M$, Leeper KJ, et al: An 18 year study of the use of beef thyrotropin to increase ${ }^{131} I$ uptake in metastatic thyroid cancer. $J$ Nucl Med 1964: 5:796-801.

11. Hays ME, Solomon DH, Pierce JG, et al: The effect of purified bovine thyroid-stimulating hormone in man: II. Loss of effectiveness with prolonged administration. $J$ Clin Endocrinol Metab 1961; 21:1475-1482.

12. Kirkpatrick $\mathrm{CH}$, Meek JC, Rich RR: Mechanism of allergy components of commercial bovine thyrotropin. $J$ Allergy Clin Immunol 1973; 51:296.

13. Krishnamurthy GT: Human reaction to bovine TSH. $J$ Nucl Med 1978; 19:284.

14. Wyngaarden JB, Wright BM, Ways R: The effect of cer- tain anions upon the accumulation and retention of iodide by the thyroid gland. Endocrinology 1952; 50:537-549.

15. Thomas ID, Odie TH, Myhill J: A diagnostic radioiodine uptake test in patients receiving antithyroid drugs. $J$ Clin Endocrinol Metab 1960; 20:1601.

16. McGirr EM, Hutchison JH, Clement WE: Sporadic goitrous cretinism. Dehalogenase deficiency in the thyroid gland of a goitrous cretin and in heterozygous carriers. Lancet 1959; 2:823.

17. Moses DC, Schteingart DE, Sturman MF, et al: Efficacy of radiocholesterol imaging of the adrenal glands in Cushing's syndrome. Surg Gynecol Obstet 1974; 139:201.

18. Thrall JH, Freitas JE, Beierwaltes WH: Adrenal scintigraphy. Semin Nucl Med 1978; 8:23-41.

19. Conn JW, Beierwaltes WH, Leiberman LM, et al: Primary aldosteronism: Preoperative tumor visualization by scintillation scanning. Endocrinology 1971; 89:713716 .

20. Conn JW, Cohen EL, Herwig KR: The dexamethasonemodified adrenal scintiscan in hyporeninemic aldosteronism (tumor vs hyperplasia). A comparison with adrenal venography and adrenal venous aldosterone. $J$ Lab Clin Med 1976; 88:841.

21. Gross MD, Valk TW, Swanson DP, et al: The role of pharmacologic manipulation in adrenal cortical scintigraphy. Semin Nucl Med 1981; 11:128.

22. Gross MD, Freitas JE, Swanson DP, et al: The normal dexamethasone suppression adrenal scintiscan. $J$ Nucl Med 1979; 20:1131.

23. Freitas JE, Grekin $\mathbf{R J}$, Thrall $\mathbf{J H}$, et al: Adrenal imaging with iodomethyl-norcholesterol (I-131) in primary aldosteronism. J Nucl Med 1979; 20:7.

24. Freitas JE, Beierwaltes WH, Nishiyama R: Adrenal hyperandrogenism: Detection by adrenal scintigraphy. $J$ Éndocrinol Invest 1978; 1:59.

25. Whitaker RH: Methods of assessing obstruction in dilated ureters. Br J Urol 1973; 45:15-22.

26. Whitaker RH: Equivocal pelviureteric junction obstruction. Br J Urol 1976; 47:377-385.

27. Whitaker RH: An evaluation of $\mathbf{1 7 0}$ diagnostic pressure flow studies in the upper urinary tract. $J$ Urol 1979 121:602-604.

28. Whitaker RH: The Whitaker test. Urol Clin North Am 1979; 6.529-539.

29. Whitfield HN, Harrison NW, Sherwood T, et al: Upper urinary tract obstruction: Pressure flow studies in children. Br J Urol 1976; 48:427.

30. Jaffe RB, Middleton AW $\mathrm{J}_{\Gamma}$ : Whitaker test: Differentiation of obstruction from nonobstructive uropathy. $A J R$ 1980; 134:9-15.

31. Newhouse JH, Pfister RC: Percutaneous ureteral perfusion, in Athenasoulis CA, Pfister $\mathrm{KC}$, Greene $\mathrm{RE}$, et al (eds): Interventional Radiology. Philadelphia, WB Saunders Co, 1982, pp 455-461.

32. Rado JP, Banos C, Tako J, et al: Renographic studies during furosemide diuresis in partial ureteral obstruction. Radiol Clin 1969; 38:132.

33. O'Reilly PH, Testa HJ, Lawson RS, et al: Diuresis renography in equivocal urinary tract obstruction. $\mathrm{Br} J$ Urol 1978; 50:76-80.

34. O'Reilly PH, Lawson RS, Shields RA, et al: Idiopathic hydronephrosis-The diuresis renogram: A new noninvasive method of assessing equivocal pelviureteral junction obstruction. $J$ Urol 1979; 121:153-155.

35. Koff SA, Thrall JH, Keyes JW Jr: Diuretic radionuclide 
urography: A noninvasive method for evaluating nephroureteral dilatation. $J$ Urol $1979 ; 122: 451-454$.

36. Koff SA, Thrall JH, Keyes JW Jr: Assessment of hydroureteronephrosis in children using diuretic radionuclide urography. $J$ Urol 1980; 123:531-534.

37. Thrall JH, Koff SA, Keyes JW Jr: Diuretic radionuclide urography in the differential diagnosis of hydroureteronephrosis. Semin Nucl Med 1981; 11:89-114.

38. Koff SA, Kogan B, Kass EJ, et al: Early post-operative assessment of the functional patency of the ureterovesical junction following ureteroneocystostomy. $J$ Urol 1981; 125:554.

39. MacGregor RJ, Konnak JW, Thrall JH, et al: Diuretic radionuclide urography in the diagnosis of suspected ureteral obstruction following renal transplantation. $J$ Urol 1983; 129:710-798.

40. Conway JJ, Belman AB, King I.R, ef al: Direct and indirect radionuclide cystography. J Urol 1975; 113:689.

41. Conway JJ, Kraglik GD: Effectiveness of direct and indirect radionuclide cystography in detecting vesicoureteral reflux. $J$ Nucl Med 1976; 17:81

42. Willi U, Treves S: Radionuclide voiding cystography, in Spencer RP (ed): Interventional Nuclear Medicine. Orlando, Fla, Grune \& Stratton, Inc, 1984, pp 109-135.

43. Bower G, Lovegrove FT, Geijsel H, et al: Comparison of "direct" and "indirect" radionuclide cystography. $J$ Nucl Med 1985; $26: 465$.

44. Hricik DE, Browning PJ, Kopelman R, et al: Captoprilinduced functional renal insufficiency in patients with bilateral renal-artery stenosis or renal-artery stenosis in a solitary kidney. $N$ Engl J Med 1983; 308:373.

45. Curtis JJ, Luke KG, Whelchel JD, et al: Inhibition of angiotensin-converting enzyme in renal-transplant recipients with hypertension. $N$ Engl J Med 1983; 308:377.

46. Geyskes GG, Oei HY, Puylaert CD, et al: Renography with captopril. Changes in a patient with hypertension and unilateral renal artery stenosis. Arch Intern Med 1986; 14:1705.

47. Dubovsky EV, Curtis JJ, Luke RG, et al: Captopril test as predictor of curable hypertension in renal transplant recipients (abstract). J Nucl Med 1986; $27: 576$.

48. Nally JV, Clarke HS, Gupta BK, et al: Captopril renography in two kidney and one kidney Goldblatt hypertension in dogs. $J$ Nucl Med 1987; 28:1171.

49. Muller FB, Sealey IF, Case DB, et al: The captopril test for identifying renovascular disease in hypertensive patients. Am J Med 1986; 80:633.

50. Sfakianakis GN, Bourgoigne VJ, Jaffe D, et al: Singledose captopril scintigraphy in the diagnosis of renovascular hypertension. J Nucl Med 1987; 28:1383.

51. Strauss HW, Harrison K, Langan $K$, et al: Thallium-201 for myocardial imaging: Relation of thallium-201 to regional myocardial perfusion. Circulation 1975; 51:641.

52. Rothendler JA, Okada RD, Wilson RA, et al: Effects of a delay in commencing imaging on the ability to detect transient thallium defects. $J$ Nucl Med 1985; $26: 880$.

53. Beller GA, Watson DD, Ackell $P$, et al: Time course of thallium-201 distribution after transient myocardial ischemia. Circulation 1980; 61:791.

54. Tamaki N, Mukai $T$, Ishii $Y$, et al: Comparative study of thallium emission myocardial tomography with $180^{\circ}$ and $360^{\circ}$ data collection. . N Nucl Med 1982; $23: 661$.

55. Eisner RL, Nowak DJ, Pettigrew RI, et al: Fundamentals of $180^{\circ}$ acquisition and reconstruction in SPECT imaging. $J$ Nucl Med 1986; 27:1717.

56. Pohost GM, Zir LM, Moore RH, et al: Differentiation of transiently ischemic from infarcted myocardium by serial imaging after a single dose of thallium-201. Circulation $1977 ; 55: 294$.

57. Wackers FJT, Fetterman RC, Mattera JA, et al: Quantitative planar thallium-201 stress scintigraphy: $\Lambda$ critical evaluation of the method. Semin Nucl Med 1985 $15: 46$.

58. Garcia E, Maddahi J, Berman D, et al: Space/time quantitation of thallium-201 myocardial scintigraphy. $J$ Nucl Med 1982; 22:309.

59. Hecht HS, Hopkins JM, Rose JG, et al: Reverse redistribution: Worsening of thallium-201 myocardial images from exercise to redistribution. Radiology 1981 140:177.

60. Silberstein EB, DeVries DF: Reverse redistribution phenomenon in Thallium-201 stress tests: Angiographic correlation and clinical significance. $J$ Nucl Med 1985 26:707.

61. Boucher CA, Zir LM, Beller GA, et al: Increased lung uptake of thallium-201 during exercise myocardial imaging: Clinical hemodynamic and angiographic implications in paticnts with coronary artery disease. $\Lambda \mathrm{m} \mathrm{J}$ Cardiol 1980; 46:189.

62. Homma S, Sanjiv K, Boucher CA: Correlates of lung heart ratio of thallium-201 in coronary artery disease. J Nucl Med 1987; 28:1531.

63. Butvinick EH, Taradash MR, Shames DM, et al: Thallium-201 myocardial perfusion scintigraphy for the clinical clarification of normal, abnormal and equivocal electrocardiographic stress tests. Am J Cardiol 1978; $41: 43$.

64. Dash $H$, Massie BM, Botvinick E, et al: The noninvasive identification of left main and three-vessel coronary artery disease by myocardial stress perfusion scintigraphy and treadmill exercise electrocardiography. Circulation 1979; 60:276.

65. Massie BM, Botvinick EH, Brundage $\mathrm{BH}$ : Correlation of thallium-201 scintigrams with coronary anatomy: Factors affecting region by region sensitivity. Am $J$ Cardiol $1979 ; 44: 616$.

66. Deppo J, Yipintsoi T, Blankstein R, et al: Thallium-201 myocardial scintigraphy in patients with triple-vessel disease and ischemic exercise stress tests. Circulation 1979; 59:714.

67. McCarthy DM, Blood DK, Sclacca RR, et al: Single dose myocardial perfusion imaging with thallium-201: Application in patients with non-diagnostic electrocardiographic stress tests. $A m J$ Cardiol 1979; 43:899.

68. Rigo P, Becker LC, Griffith LSL, et al: Influence of coronary collateral vessels on the results of thallium-201 myocardial stress imaging. Am $J$ Cardiol 1979; 44:452.

69. Ritchie JL, Zaret BL, Strauss HW, et al: Myocardial imaging with thallium-201: A multicenter study in patients with angina pectoris or acute myocardial infarction. Am $J$ Cardiol 1978; 42:345.

70. Meller J, Goldsmith SJ, Rudin A, et al: Spectrum of exercise thallium-201 myocardial perfusion imaging in patients with chest pain and normal coronary angiograms. Am J Cardiol 1979; 43:717.

71. Turner DA, Battle WE, Deshmukh $\mathrm{H}$, et al: The predictive value of myocardial perfusion scintigraphy after stress in patients without previous myocardial infarction. I Nucl Med 1978; 19:249.

72. Okada RD, Boucher CA, Strauss HW, et al: Exercise radionuclide imaging approaches to coronary artery disease. Am J Cardiol 1980; 46:1188.

73. Vogel RA, Kirch DL, LeFree M'T, et al: Thallium-201 
myocardial perfusion scintigraphy: Results of standard and multipinhole tomographic technique. Am $J$ Cardiol 1979; 43:787.

74. Rizi HR, Kline RC, Thrall JH, et al: Thallium-201 myocardial scintigraphy: A critical comparison of sevenpinhole tomography and conventional planar imaging. I Nucl Med 1981; 22:493

75. Tamaki $N$, Makai $T$, Ischii $Y$, et al: Clinical evaluation of thallium-201 emission myocardial tomography using a rotating gamma camera: Comparison with sevenpinhole tomography. $J$ Nucl Med 1981; $22: 849$.

76. Maublant J, Cassaghess J, Jourde $M$, et al: Myocardial emission tomography with thallium-201. Eur $J$ Nucl Med 1981; 6:289.

77. Burow RD, Pond M, Schafer AW, et al: "Circumferential profiles": A new method for computer analysis of thallium-201 myocardial perfusion images. $J$ Nucl Med $1979 ; 20: 771$.

78. Meade RC, Bameah VS, Horgan JD, et al: Quantitative methods in the evaluation of thallium-201 myocardial perfusion images. $J$ Nucl Med 1978; 19:1175.

79. Berger BC, Watson DD, Taylor GJ, et al: Quantitative thallium-201 exercise scintigraphy for detection of coronary artery disease. $J$ Nucl Med $1981 ; 22: 585$.

80. Garcia EV, VanTrain K, Maddahi J, et al: Quantification of rotational thallium-201 myocardial tomography. $J$ Nucl Med 1985; 26:17.

81. Watson DD, Campbell NP, Read EK, et al: Spatial and temporal quantitation of planar thallium myocardial images. J Nucl Med 1981; 22:577.

82. Eisner $h$, Churvhwell A, Noever $T$, el al: Quantitative analysis of the tomographic thallium-201 myocardial bullseye display: Critical role of correcting for patient motion. J Nucl Med 1988; 29:91.

83. VanTrain K, Berman DS, Garcia EV, et al: Quantitative analysis of stress thallium-201 myocardial scintigrams: A multicenter trial. $J$ Nucl Med 1986; $27: 17$.

84. Diamond GA, Forrester JS: $\Lambda$ nalysis of probability as an aid in the clinical diagnosis of coronary artery disease. $N$ Engl J Med 1979; 300:1350.

85. Hamilton GW, Trobaugh GB, Ritchie JL, et al: Myocardial imaging with thallium-201: An analysis of clinical usefulness of Bayes' theorem. Semin Nucl Med 1978; 8:358.

86. Albro PC, Gould KL, Westcott $\mathrm{RJ}$, et al: Noninvasive assessment of coronary stenoses by myocardial imaging during pharmacologic coronary vasodilatation. III. Clinical trial. Am $J$ Cardiol 1978; $42: 751$.

87. Leppo J, Boucher CA, Okada RD, et al: Serial thallium201 nyocardial inaging after dipyridanole infusion: Diagnostic utility in detecting coronary stenosis and relationship to regional wall motion. Circulation 1982; $66: 649$.

88. Haynie MP, Gould KL, Gerson MC: Methods alternative to dynamic leg exercise for detecting chronic coronary artery disease, in Gerson MC, (ed): Cardiac Nuclear Mcdicine. New York, McGraw Hill International Book Co, 1987, pp 235-250.

89. Gould KL, Sorenson SG, Albro P, et al: Thallium-201 myocardial imaging during coronary vasodilation induced by oral dipyridamole. $J$ Nucl Med 1986; $27: 31$.

yo. Borer IS, Bacharach SL, Green MV, et al: Realtime radionuclide cineangiography in the non-invasive evaluation of global and regional left ventricular function at rest and during exercise in patients with coronary artery disease. $N$ Engl $J$ Med 1977; 296839.

91. Jones RH, McEwan P, Newman G, et al: The accuracy of diagnosis of coronary artery disease by radionuclide measurements of left ventricular function during rest and exercise. Circulation 1981; 64:586.

92. Berger HJ, Reduta LA, Johnston DE, et al: Global and regional left ventricular response to bicycle exercise in coronary artery disease: Assessment by quantitative radionuclide angiocardiography. Am $J$ Med 1979; 66:13.

93. Borer JS, Kent KM, Bacharach SL, et al: Sensitivity, specificity and predictive accuracy of radionuclide cineangiography during exercise in patients with coronary artery disease: Comparison with exercise electrocardiography. Circulation 1979; 60:572.

94. Brady TJ, Thrall JH, Pitt B: The importance of adequate exercise in the detection of coronary heart disease by radionuclide ventriculography. $J$ Nucl Med $1980 ; 21: 1125$.

95. Caldwell JH, Hamilton GW, Sorenson SG, et al: The detection of coronary artery disease with radionuclide techniques: A comparison of rest-exercise thallium imaging and ejection fraction response. Circulation 1980; 61:610.

96. Bodenheimer MM, Banka VS, Fooshee CM, et al: Detection of coronary heart disease using radionuclide determined regional ejection fraction at rest and during grip exercise: Correlation with coronary arteriography. Circulation 1978; 58:640.

97. Peter CA, Jones RH: Effects of isometric handgrip and dynamic exercise on left ventricular function. $J$ Nucl Med 1980; 21:1131.

98. Manyari DE, Nolewajka AJ, Purves P, et al: Comparative value of the cold-pressor test and supine bicycle exercise to detect subjects with coronary artery disease using radionuclide ventriculography. Circulation 1982; 65:571-579.

99. Jordan LJ, Boner JS, Zullo $M$, et al: Exercise versus cold temperature stimulation during radionuclide cincangiography: Diagnostic accuracy in coronary artery disease. Am J Cardiol 1983; 51:1091.

100. Dehmer GJ, Firth BG, Nicod P, et al: Alterations in left ventricular volumes and ejection fraction during atrial pacing in patients with coronary artery disease. Assessment with radionuclide ventriculography. Am Heart $J$ 1983; 106:114.

101. Dyke SH, Cohn PF, Gorkin R, et al: Detection of residual myocardial function in coronary artery disease using post-extra-systolic potentiation. Circulation 1974; 50:694.

102. Kalff V, Chan W, Rabinovitch $M$, et al: Radionuclide evaluation of postextrasystolic potentiation of left ventricular function induced by atrial and ventricular stimulation. Am J Cardiol 1982; 50:106.

103. Malmud LS, Fisher RS: Radionuclide studies of esophageal transit and gastroesophageal reflux. Semin Nucl Med 1982; 12:104.

104. Fisher RS, Malmud LS, Roberts GS, et al: Gastroesophageal (GE) scintiscanning to detect and quantitate GE reflux. Gastroenterology 1976; 70:301.

105. Malmud LS, Fisher RS: Gastroesophageal scintigraphy. Gastrointest Radiol 1980; 5:195.

106. Malmud LS, Charkes ND, Littlefield J, et al: The mode of action of alginic acid compound in the reduction of gastroesophageal reflux. J Nucl Med 1979; 20:1023.

107. Malmud LS, Fisher RS, Knight LC, et al: Scintigraphic evaluation of gastric emptying. Semin Nucl Med 1982; 12:116.

108. Shapiro B, Gross MD: Interventional studies of the up- 
per gastrointestinal tract, in Thrall JH, Swanson DP (eds): Diagnostic Interventions in Nuclear Medicine. Chicago, Year Book Medical Publishers, Inc, 1985, pp $204-225$.

109. Meyer JH, MacGregor MD, Gueller R, et al: Tc-99m tagged chicken liver as a marker of solid food in the human stomach. Digest Dis 1976; 21:296.

110. Domstad PA, Kim EE, Coupal JJ, et al: Biological gastric emptying time in diabetic patients using Tc-99m labeled resin oatmeal with and without metoclopramide. J Nucl Med 1980; 21:1098.

111. Sfakianakis GN, Conway JJ: Detection of ectopic gastric mucosa in Meckel's diverticulum and in other aberrations by scintigraphy: I. Pathophysiology and 10year clinical experiences. $J$ Nucl Med 1981; 22:697.

112. Sfakianakis GN, Conway JJ: Detection of ectopic gastric mucosa in Meckel's diverticulum and in other aberrations by scintigraphy: II. Indications and methodsA 10-year experience. J Nucl Med 1981; 22:732.

113. Khettery J, Effmann E, Grand RJ, et al: Effects of pentagastrin, histalog, glucagon, secretin, and perchlorate on the gastric handling of ${ }^{99 \mathrm{~m}} \mathrm{Tc}$ pertechuetate in mice. Radiology 1976; 120:629.

114. Sfakianakis GN, Anderson GF, King OR, et al: The effect of gastrointestinal hormones on the pertechnetate imaging of ectopic mucosa in experimental Meckel's diverticulum. $J$ Nucl Med 1981; 22:678.

115. Treves S, Grand RJ, Eraklis AJ: Pentagastrin stimulation of technetium-99m uptake by ectopic gastric mucosa in a Meckel's diverticulum. Radiology 1978; 128:711.

116. Froelich JW, Juni JE: Intravenous glucagon as an adjuvant for diagnosing bleeding of the small bowel with in-vivo Tc-99m labeled red blood cells. Radiology 1984; 151:239.

117. Yaffe SJ, Juchau MR: Perinatal pharmacology. Annu Rev Pharmacol 1974; 14:219.

118. Thaler MM: Effect of phenobarbital on hepatic transport and excretion of ${ }^{131}$ I-rose bengal in children with cholestasis. Pediatr Res 1972; 6:100.

119. Majd M, Reba RC, Altman RP: Hepatobiliary scintigraphy with ${ }^{99 \mathrm{~m}}$ Tc-PIPIDA in the evaluation of neonatal jaundice. Pediatrics 1981; 67:140.

120. Majd $M$, Reba $\mathbf{h}$, Altman RP: Effect of phenobarbital on ${ }^{99 m}$ Tc-IDA scintigraphy in the evaluation of neonatal jaundice. Semin Nucl Med 1981; 11:194.

121. Weissman HS, Badia JS, Sugarman LA, et al: Spectrum of ${ }^{99 \mathrm{~m}} \mathrm{Tc}$-IDA cholescintigraphic patterns in acute cholecystitis. Radiology 1981; 138:167.

122. Freitas JE: Cholescintigraphy in acute and chronic cholecystitis. Semin Nucl Med 1982; 12:18.
123. Freitas JE, Fink-Bennett DM, Thrall JH, et al: Efficacy of hepatobiliary imaging in acute abdominal pain. $J$ Nucl Med 1980; 21:919.

124. Kalff $V$, Froelich JW, Lloyd $R$, et al: The reduced predictive value of an abnormal hepatobiliary scan in a patient with severe intercurrent illness. Radiology $1983 ; 146: 191$.

125. Freeman LM, Sugarman LA, Weissman HS: Role of cholecystokinetic agents in Tc-99m IDA cholescintigraphy. Semin Nucl Med 1981; 11:186.

126. Choy D, Shi EC, McLean RG, et al: Cholescintigraphy in acute cholecystitis: Use of intravenous morphinc. Radiology 1984; 151:203.

127. Fink-Bennett D, DeRidder P, Kolozsk $W$, et al: Cholecystokinin cholescintigraphic findings in the cystic duct syndrome. $J$ Nucl Med 1985; 26:1123.

128. Cozzolino JH, Goldstein F, Greening PR, et al: The cystic duct syndrome. JAMA 1963; 185:100.

129. Sokoloff L, Reivich $M$, Kennedy $C$, et al: The $\left({ }^{1+} \mathrm{C}\right)$ deoxyglucose method for the measurement of local cerebral glucose utilization: Theory, procedure and normal values in the conscious and anesthetized albino rat. $J$ Neurochem $1977 ; 28: 897$.

130. Reivich M, Kuhl D, Wolf A, et al: The $\left({ }^{18} F\right)$ flunrodenxyglucose method for the measurement of local cerebral glucose utilization in man. Circ Res 1979; 44:127.

131. Phelps ME, Huang SC, Hoffman EJ, et al: Tomographic measurement of local cerebral glucose metabolic rate in humans with (F-18) 2-fluoro-2-deoxyglucose: Validation of method. Ann Neurol 1979; 6:371.

132. Phelps ME, Kuhl DE, Mazziotta JC: Metabolic mapping of the brain's response to visual stimulation: Studies in humans. Science 1981; 211:1445.

133. Mazziotta JC, Phelps ME, Miller J, et al: Tomographic mapping of human cerebral metabolism: Normal unstimulated state. Neurology (NY) 1981; 31:503.

134. Phelps ME, Mazziotta JC, Kuhl DE, et al: Tomographic mapping of human cerebral metabolism: Visual stimulation and deprivation. Neurology $(N Y)$ 1981; 31:517.

135. Mazziotta JC, Phelps ME, Carson RE, et al: Tomographic mapping of human cerebral metabolism: Auditory stimulation. Neurology (NY) 1982; 32:921.

136. Wagner HN Jr, Burns HD, Dannals RF, et al: Imaging dopamine receptors in the human brain by positron tomography. Science 1983; 221:1264.

137. Frost JJ, Wagner HN Jr, Dannals $\mathrm{RF}$, et al: Imaging op iate receptors in the human brain by positron tomography. J Comput Assist Tomogr 1985; 9:231. 